\title{
NK Cell Priming From Endogenous Homeostatic Signals Is Modulated by CIS
}

\author{
Rebecca B. Delconte ${ }^{1,2}$, Geoffrey Guittard ${ }^{3}$, Wilford Goh ${ }^{1,2}$, Soroor Hediyeh-Zadeh ${ }^{2,4}$, \\ Robert J. Hennessy ${ }^{1,2}$, Jai Rautela ${ }^{5,6}$, Melissa J. Davis ${ }^{2,4}$, \\ Fernando Souza-Fonseca-Guimaraes ${ }^{7}$, Jacques A. Nunès ${ }^{3}$ and \\ Nicholas D. Huntington ${ }^{1,2,5,6 *}$
}

1 Division of Molecular Immunology, The Walter and Eliza Hall Institute of Medical Research, Parkville, VIC, Australia, ${ }^{2}$ Department of Medical Biology, Faculty of Medicine, Dentistry and Health Sciences, University of Melbourne, Melbourne, VIC, Australia, ${ }^{3}$ Centre de Recherche en Cancérologie de Marseille, CRCM, Immunity and Cancer Team, Institut Paoli-Calmettes, Inserm, CNRS, Aix Marseille Université, Marseille, France, ${ }^{4}$ Division of Bioinformatics, The Walter and Eliza Hall Institute of Medical Research, Parkville, VIC, Australia, ${ }^{5}$ oNKo-Innate Pty Ltd., Melbourne, VIC, Australia, ${ }^{6}$ Department of Biochemistry and Molecular Biology, Biomedicine Discovery Institute, Monash University, Clayton, VIC, Australia, ${ }^{7}$ University of Queensland Diamantina Institute, Translational Research Institute, University of Queensland, Brisbane, QLD, Australia

OPEN ACCESS

Edited by:

Thierry Walzer,

UMR5308 Centre International de Recherche en Infectiologie

(CIRI), France

Reviewed by:

Subramaniam Malarkannan, Medical College of Wisconsin, United States

Hugh J. M. Brady,

Imperial College London,

United Kingdom

*Correspondence:

Nicholas D. Huntington

nicholas.huntington@monash.edu

Specialty section:

This article was submitted to

NK and Innate Lymphoid Cell Biology,

a section of the journal

Frontiers in Immunology

Received: 05 November 2019

Accepted: 13 January 2020

Published: 31 January 2020

Citation:

Delconte RB, Guittard G, Goh W, Hediyeh-Zadeh S, Hennessy RJ,

Rautela J, Davis MJ,

Souza-Fonseca-Guimaraes F, Nunès JA and Huntington ND (2020)

NK Cell Priming From Endogenous Homeostatic Signals Is Modulated by

CIS. Front. Immunol. 11:75

doi: 10.3389/fimmu.2020.00075
Natural killer (NK) cell activation is controlled by a balance of activating and inhibitory signals and cytokines such as IL-15. We previously identified cytokine-inducible $\mathrm{SH} 2$-containing protein (CIS) as a negative regulator of $\mathrm{IL}-15$ signaling in NK cells under inflammatory conditions. While the functional effect of Cish-deficiency in NK cells was obvious by their increased anti-tumor immunity and hyper-proliferative response to IL-15, it remained unclear how CIS regulates NK cell biology in steady-state. Here, we investigated the role of CIS in the homeostatic maintenance of NK cells and found CIS-ablation promoted terminal differentiation of NK cells and increased turnover, suggesting that under steady-state conditions, CIS plays a role in maintaining IL-15 driven regulation of NK cells in vivo. However, hyper-responsiveness to IL-15 did not manifest in NK cell accumulation, even when the essential NK cell apoptosis mediator, Bc/2/11 (BIM) was deleted in addition to Cish. Instead, loss of CIS conferred a lower activation threshold, evidenced by augmented functionality on a per cell basis both in vitro and in vivo without prior priming. We conclude that Cish regulates IL-15 signaling in NK cells in vivo, and through the rewiring of several activation pathways leads to a reduction in activation threshold, decreasing the requirement for priming and improving NK cell anti-tumor function. Furthermore, this study highlights the tight regulation of NK cell homeostasis by several pathways which prevent NK cell accumulation when IL-15 signaling and intrinsic apoptosis are dysregulated.

Keywords: NK cell, CIS, priming, Homeostasis, IL-15

\section{INTRODUCTION}

NK cells are bone marrow (BM) derived lymphocytes that circulate through blood and lymphoid tissues, acting as sentinels of the immune system (1). Their role in protection against cancer has been explored and studies have found increased incidence of cancer in mice and humans that lack NK cells or possess reduced NK cell cytotoxicity (2-4). In addition, a growing number of clinical 
trials have emerged harnessing the anti-tumor potential of NK cells. It is increasingly evident that NK cell therapies are advantageous over $\mathrm{T}$ cell therapies in the clinic in terms of their safety profile (5). Utilizing NK cells in clinical settings such as adoptive cell therapy and chimeric antigen receptor (CAR) engineered cells reduces the incidence of severe cytokine release syndrome (CRS) and graft-versus-host disease (GvHD) side effects (6-8). Improving patient outcomes without contributing to severe side-effects have been the driving force behind improving $\mathrm{NK}$ cell immunotherapies and as such, a more comprehensive understanding of the mechanisms regulating NK cell homeostasis have indefinite clinical implications.

We recently identified cytokine-inducible SH2-containing protein (CIS, encoded by Cish) as a critical intracellular immune checkpoint in NK cells (9). Indeed, loss of CIS promotes NK cell anti-tumor functions and represents a novel strategy to improve immunotherapy. However, we still do not fully appreciate mechanistically how CIS regulates NK cell anti-tumor functions. We previously showed that CIS regulates IL-15 signaling in NK cells (9). IL-15 is the main driver of NK cell proliferation, survival, differentiation and function, and thus is highly relevant in NK cell homeostasis $(10,11)$. While the functional effect of Cish-deficiency in NK cells was obvious by their increased antitumor immunity and hyper-proliferative response to IL-15, it remained unclear how CIS regulated NK cell biology in vivo during steady-state.

Whilst NK cells differ from other adaptive immune cells in that they do not require pre-exposure to an antigen to elicit a rapid immune response, there is accumulating evidence implicating the importance of $\mathrm{NK}$ cell priming by various cytokines and factors to achieve their maximum response and full repertoire of effector functions. These include IL-15 transpresentation by dendritic cells (12) or macrophages (13), IL18 (14), IL-12 (15, 16), and Type 1 IFN (17). These data advocate for the notion that there is only a very small fraction of NK cells in vivo that qualify as fully-fledged effector cells in vitro, and it is likely that these are cells that have been exposed to in vivo priming. It would suffice to say then, that NK cell priming can reduce the activation threshold required to elicit a full, directed cytotoxic response toward an infected or cancerous cell. Thus, identifying factors that may reduce this threshold is an important aspect of NK cell biology with the potential to improve NK cell fitness and immunotherapy potential.

To further our understanding of NK cell regulation, we investigated the role of CIS in the homeostatic maintenance of NK cell numbers in vivo and evaluated the impact of IL15 signaling in steady state. In Cish-deficient mice (germline, $\mathrm{Cish}^{-1-}$ ), the terminal differentiation of NK cells is promoted, as well as increased expression of cell cycle markers. This suggests that even under steady-state conditions, CIS plays a role in maintaining IL-15 driven regulation of NK cells in vivo. This homeostatic alteration did not manifest in an accumulation of CIS-deficient NK cells, instead, loss of CIS conferred a lower activation threshold. Global gene expression of $\mathrm{Cish}^{-/-} \mathrm{NK}$ cells compared to WT NK cells revealed upregulation of several signaling pathways and genes important for NK cell function. We conclude that the increased anti-tumor function observed in Cish-deficient mice is not caused by homeostatic alterations in, or accumulation of NK cells, but rather the increased anti-tumor effect is a direct consequence of the rewiring of several activation and signaling pathways that results in a reduced activation threshold and decreased requirement for priming.

\section{RESULTS}

\section{Cish-Deficient Mice Have More Terminally Differentiated NK Cells}

NK cells with a germline deletion of the Cish gene are hyperresponsive to IL-15 due to a lack of receptor signaling dampening (9). Stimulation with both pro-survival and mitogenic concentrations of IL-15 (5 and $\geq 10 \mathrm{ng} / \mathrm{ml}$, respectively) induced enhanced proliferation of Cish-deficient NK cells when compared to WT NK cells (Figure 1A). The increased proliferation of Cish-deficient NK cells was analogous to increased survival over time, resulting in increased total cell numbers (Supplemental Figure 1A). Despite the robust increase in proliferation and survival in vitro, under homeostatic conditions, germline $\mathrm{Cish}^{-/-}$mice have normal numbers and frequencies of peripheral NK cells in the blood and all lymphoid organs (Supplemental Figure 1B). We next sought to look at NK cell progenitors in the BM. Previously published RNA sequencing analysis of NK cell progenitors show that NK cell precursors $\left(\mathrm{CD} 122^{+} \mathrm{IL}_{\mathrm{R}}{ }^{+} \mathrm{NK} 1.1^{-} \mathrm{DX} 5^{-} \mathrm{CD} 3^{-} \mathrm{CD} 19^{-}\right)$in the $\mathrm{BM}$ express relatively higher levels of Cish than mature NK cells (9). We quantified and compared total cell numbers of NK cell-precursors and observed that they were equivalent and thus unaffected by loss of CIS (Supplemental Figure 1C). In line with previous studies, we also showed that all haematopoietic cells in Cish-deficient mice are equivalent in numbers to WT mice (Supplemental Figure 1D).

To explore why Cish-deficient NK cells appear "normal" under steady-state conditions, we analyzed the maturation stages of splenic and BM Cish ${ }^{-/-}$NK cells. NK cells expressing both NK1.1 and NKp46 mature in a linear fashion from an immature stage $\mathrm{CD}_{11 \mathrm{~b}}^{-} \mathrm{KLRG}^{-}$(Imm), through to an intermediate stage $\left(\mathrm{CD} 11 \mathrm{~b}^{+} \mathrm{KLRG1}^{-} ; \mathrm{M} 1\right)$ and finally reach full maturity with the upregulation of KLRG1 [(CD11b ${ }^{+} \mathrm{KLRG}^{+}$; M2) Figure 1B] (18). We observed a significant increase in the proportion of mature, M2 NK cells in the spleen of $\mathrm{Cish}^{-/-}$mice, and this resulted in a compensatory reduction in frequency of M1 and Imm subsets when compared to Cish ${ }^{+/+}$mice (Figure 1C). We also observed a minor increase in the M2 subset in the BM of $\mathrm{Cish}^{-/-}$mice and a compensatory reduction in the frequency of M1s. However, this was not as dramatic as what was observed in the spleen (Figure 1D). Similarly, gating using conventional markers of NK cell maturation [CD27 and CD11b $(19,20)]$ also showed an increase in $\mathrm{CD}_{27}^{-} \mathrm{CD} 11 \mathrm{~b}^{+}$mature NK cells and the subsequent decrease in $\mathrm{CD} 27^{+} \mathrm{CD} 11 \mathrm{~b}^{+}$intermediates in the spleen and BM (Supplemental Figures 1E,F). These data suggest that the requirement for CIS in controlling NK cell turnover rate and/or maturation is not conserved between the primary site of NK cell generation (BM) and peripheral organs.

To determine whether a loss of CIS manifests in the alteration of other important NK cell receptors in the periphery, we next sought to measure receptor expression on splenic $\mathrm{Cish}^{-/-}$ 
A

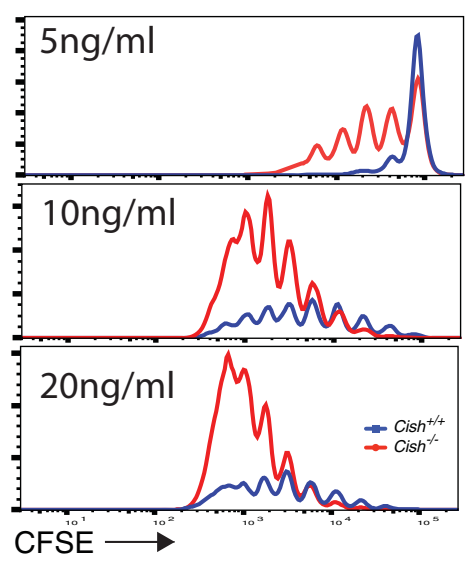

B

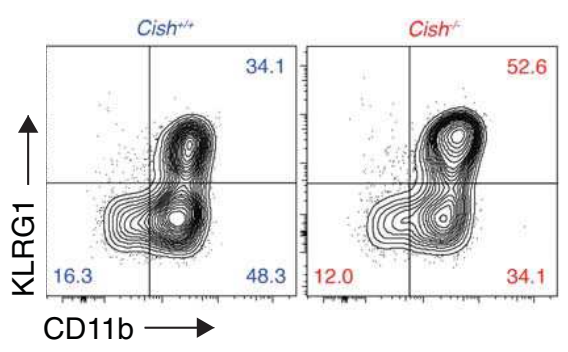

C

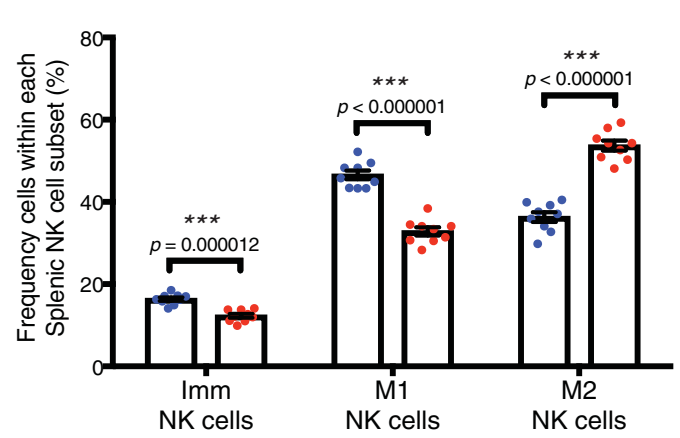

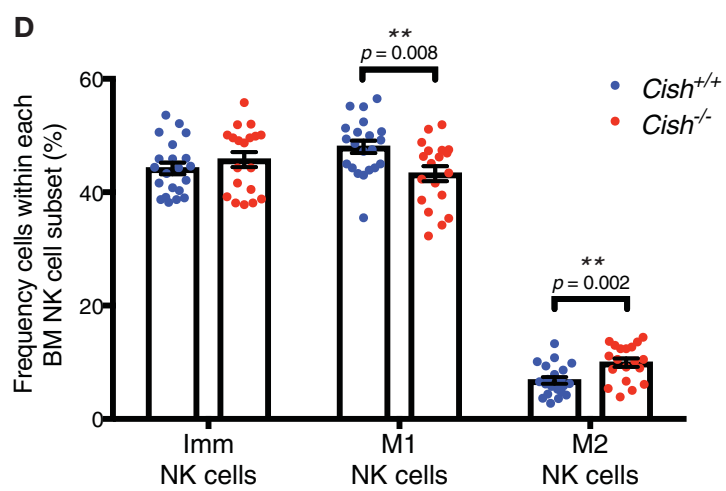

E

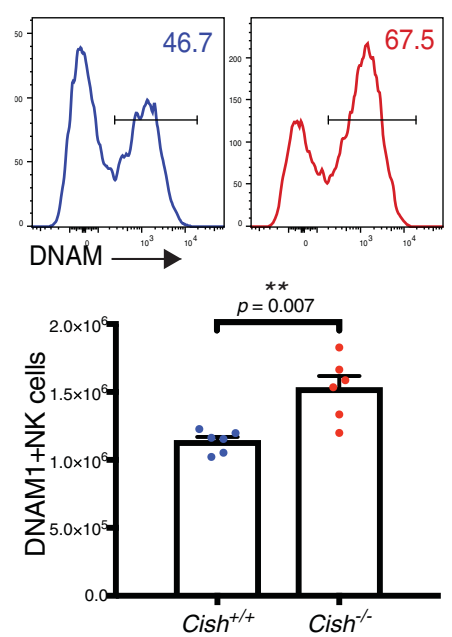

F
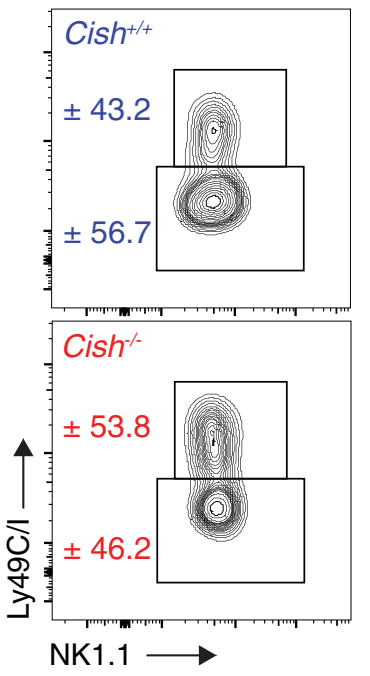

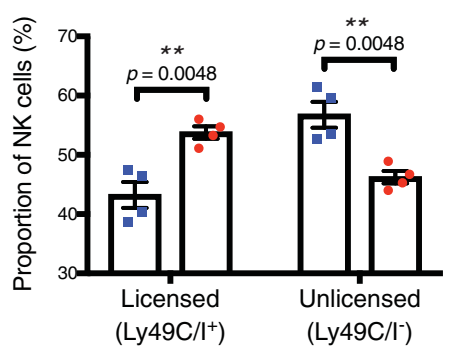

(Ly49C/I+)

(Ly49C/I)

FIGURE 1 | CIS-deficient NK cells show increased maturation. (A) Purified and CTV-labeled Cish ${ }^{-/-}$and Cish ${ }^{+/+}$NK cells were seeded at $1 \times 10^{4}$ cells/well into round wells containing different concentrations of IL-15. Cells were incubated at $37^{\circ} \mathrm{C}$ in a humidified environment containing $5 \% \mathrm{CO}$ for $240 \mathrm{~h}$. Representative histogram overlays are shown. (B-D) NK cell maturation subsets were quantified by flow cytometric analysis of KLRG1 and CD11b in Cish ${ }^{+/+}$and Cish ${ }^{-/-}$mice. (B) Representative FACS plots showing the abundance of each maturation subset and gating strategy in the spleen. Frequency of Imm, M1, and M2 cells were measured within NK cell populations from the (C) spleen and (D) BM ${ }^{\star *} p<0.01,{ }^{\star \star *} p<0.001$ (unpaired Student's $t$-test). (E,F) Flow cytometry was used to quantify DNAM-1 and Ly49C/I expression. Representative histograms of DNAM-1 expression (E, top panel) and Ly49C/I expression (F, left panel) and frequency of DNAM-1+ NK cells (E, bottom panel), and licensed and unlicensed NK cells (F, right panel) ${ }^{* *} p<0.01$ (unpaired Student's $t$-test). (A,B, one representative of three experiments; $\mathbf{C}, n=9$ biological replicates mean \pm s.e.m.; E, F, values indicate mean \pm s.e.m. and $n \geq 4$ biological replicates). 
NK cells ex vivo. We observed a significant increase in the proportion of DNAM-1 ${ }^{+} \mathrm{NK}$ cells within the $\mathrm{Cish}^{-/-} \mathrm{NK}$ cell population (Figure 1E). The increase in both DNAM-1 ${ }^{+}$ and $\mathrm{KLRG}^{+} \mathrm{NK}$ cells in Cish-deficient mice suggests that under steady state conditions, CIS plays a role in regulating the IL-15-dependent differentiation of NK cells in vivo. The preferential increase in DNAM-1 ${ }^{+} \mathrm{NK}$ cells could also be explained by our previous published data which showed an increase in IL-15 receptor responsiveness of the DNAM-1 ${ }^{+}$ proportion of NK cells in vivo (21). Furthermore, Ly49C/I receptor expression was altered in Cish-deficient NK cells compared to wildtype controls (Figure 1F). Ly49C/I expression defines NK cells into two populations of licensed $\left(\mathrm{Ly} 49 \mathrm{C} / \mathrm{I}^{+}\right)$ and unlicensed $\left(\mathrm{Ly} 49 \mathrm{C} / \mathrm{I}^{-}\right)$cells, with each subset possessing differential ability to lyse target cells and respond to infection $(22,23)$. Studies have shown that licensing is important for NK cells to acquire effector functions. As such, Ly49deficient mice harbor unlicensed $\mathrm{NK}$ cells, which display impaired recognition of MHC-I-deficient target cells and reduced cytotoxicity (24). Cish-deficient NK cells show a significant increase in their licensed proportion (Figure 1F), suggesting that they may have a greater cytotoxic capacity and/or augmented activation.

\section{CIS Governs NK Cell Turnover but Not Accumulation in vivo}

Published data suggests that $\mathrm{KLRG}^{+}$NK cells accumulate following periods of acute proliferation (18), thus we next examined whether a loss of CIS induced any changes in proliferation or turnover in vivo. Ki67 is a marker associated with cell-proliferation and its expression is absent on resting cells (G0) but is expressed during all active phases of cell cycle (G1, S, G2 and mitosis) (25). Cish ${ }^{-/-}$NK cells show a marked increase in the cell cycle marker, Ki67 in splenic NK cells (Figure 2A). Since there was an observable increase in $\mathrm{KLRG}^{+}$(M2) cells in $\mathrm{Cish}^{-1-}$ mice, we sought to understand whether the changes in cell cycle were fixed to one particular subset. Interestingly, Ki67 was increased across all stages of NK cell maturation, including the M2 subset (Figure 2B). To confirm this in vitro, the response of each maturation subset to IL-15 was assessed by labeling sorted Imm, M1, and M2 cells with CTV and stimulating them with IL15 for 5 days. Previous work from our group and others have shown that response to IL-15 signaling is decreased as NK cell maturation increases $(18,20)$. Intriguingly, while each subset showed an increase in proliferation when compared to their $\mathrm{Cish}^{+/+}$counterpart (data not shown), the proliferation profile of the maturation stages was conserved in Cish ${ }^{-/}$NK cells (Supplemental Figure 2A). Thus, M2 cells were less responsive to IL-15 than M1, and M1 less responsive than Imm. To determine whether IL-15R $\beta$ (CD122) levels could account for the proliferation profile, CD122 expression was also assessed. CD122 expression was found to be increased in the Imm, M1, and M2 subsets of $\mathrm{Cish}^{-/-}$NK cells when compared to Cish ${ }^{+/+}$NK cells (Supplemental Figure 2B). However, a loss of sensitivity to IL-15 is still observed as the Cish ${ }^{-/-}$NK cells mature. This is evidenced by the downregulation of CD122 upon KLRG1 upregulation, suggesting that regulation of CD122 is independent of CIS, but dependent on IL-15.

As there was both an accumulation of $\mathrm{KLRG}^{+}$and $\mathrm{Ki}^{+}{ }^{+}$ NK cells in Cish-deficient mice, yet no increase in cell numbers, we next asked whether the rate of death of Cish-deficient NK cells was increased. The thymidine analog, EdU, is incorporated into DNA during active DNA synthesis and can be used as a measure of cell turnover. Pulse-chase experiments using EdU are commonly used for examining cellular processes over time, thus we assessed the rate of NK cell turnover in the spleen and BM by administering EdU for a set period and subsequently measured the rate of loss of EdU over time (Figure 2C). Consistent with the increase in Ki67, we observed an increase in EdU uptake in total Cish-deficient NK cells on Day 3, indicating that CIS is involved in regulating NK cell turnover in vivo (Figure 2D). Consistent with the increase in EdU in total NK cells, both the Imm and M1 subsets of $\mathrm{Cish}^{-/-}$NK cells showed increased incorporation of EdU, as did the DNAM-1 ${ }^{+}$subset. Previous studies have shown that KLRG1 expressing NK cells are less responsive to proliferative signals in vivo (18), and so it was interesting that we observed the M2 subset of Cish-deficient NK cells to also show an increase in EdU uptake, albeit not significantly. The increase of $\mathrm{KLRG}^{+} \mathrm{NK}$ cells we observed in Cish-deficient mice, together with the reduction in Imm and M1 NK cells, and the increased uptake of EdU amongst all three maturation subsets suggests that CIS slows down the rate at which NK cells cycle through from the Imm stage to the M2 stage.

To determine whether the rate of death was increased in the absence of CIS, EdU uptake, and loss were compared over time as outlined in Figure 2C. Measuring EdU uptake over time, we found that the fold change of EdU from initial uptake (at Day 3) was actually increased in the spleen at Day 16, but decreased in the BM. However, when we measured EdU at Day 30, we found that the proportion of $\mathrm{EdU}^{+} \mathrm{Cish}^{-/-} \mathrm{NK}$ cells was decreased (Figures 2E,F, left panels). The fold change of $\mathrm{EdU}^{+} \mathrm{NK}$ cells over time also showed that the loss of EdU from the initial uptake at Day 3 was rapidly lost from $\mathrm{Cish}^{-1-} \mathrm{NK}$ cells in the BM (Figure 2F, right panel). Previous studies have showed that in mice, NK cell turnover in the BM is significantly faster than in the spleen, and that this occurs due to the requirement for precursor populations of NK cells to populate the splenic NK cell niche (26). It is possible that the increase in EdU observed in the spleen at Day 16 is actually EdU ${ }^{+} \mathrm{BM}$ NK cells that have migrated to the spleen, and that this must occur continuously during the lifespan of Cish-deficient mice due to the rapid turnover of NK cells in the spleen. The increased rate of loss of EdU in $\mathrm{Cish}^{-/} \mathrm{NK}$ cells at Day 30 in the spleen is also suggestive of their increased turnover rate and in turn, increased cell death. The rapid cell death did not result in an accumulation of dying cells, ruled out by Caspase 3/7 staining (Supplemental Figure 2C), and similarly there were no significant changes in pro- or anti-apoptotic proteins BIM, MCL1, and BCL2 (Supplemental Figure 2D). Though somewhat surprising, the rapid rate at which $\mathrm{Cish}^{-/-}$ NK cells are turning over and the subsequent dead/dying cells are cleared by macrophages and phagocytes may explain why there is no observable accumulation. Together, these data suggest that CIS slows down the rate of NK cell turnover and cell 


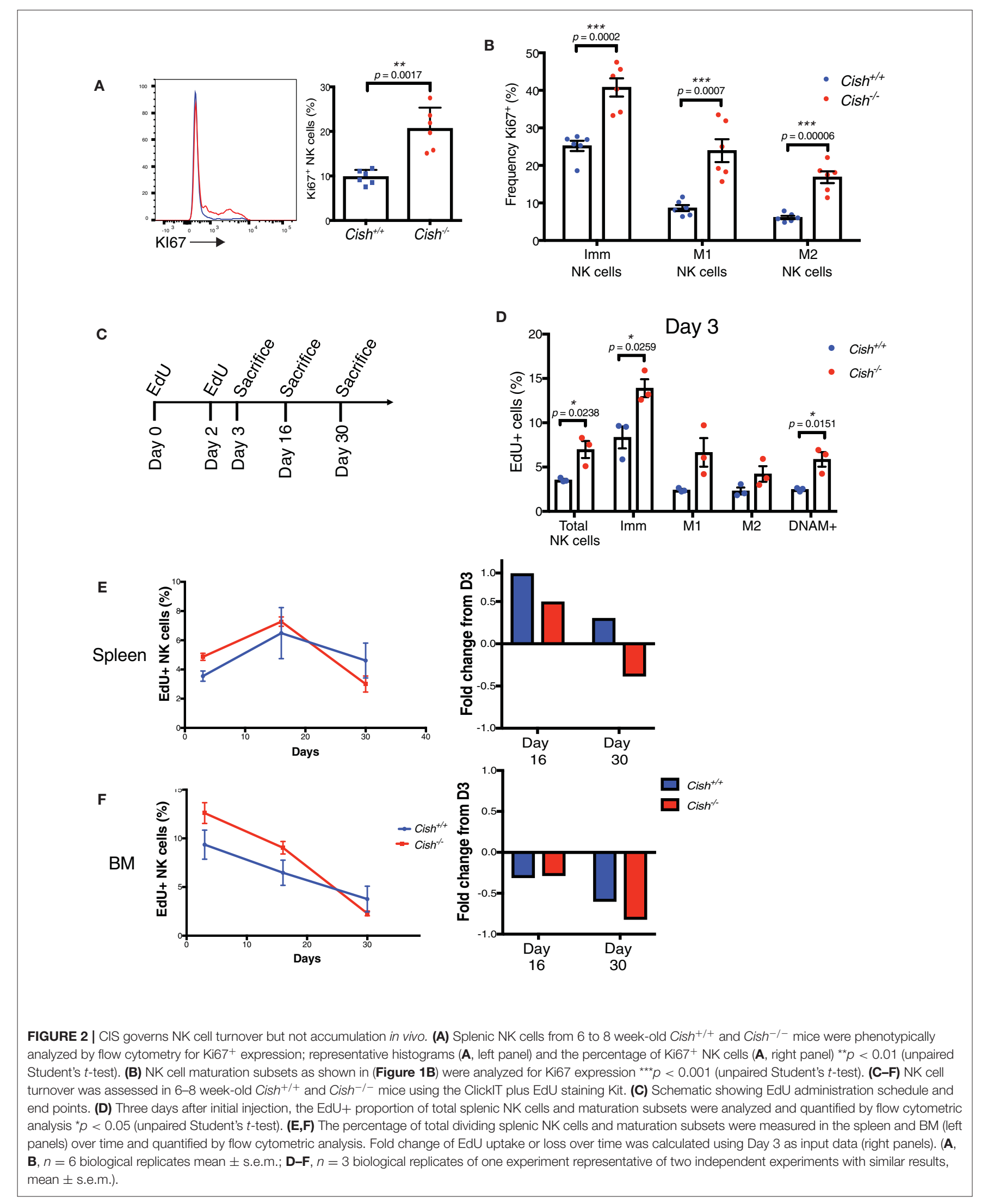


death, limiting the responsiveness of NK cells to IL-15 under homeostatic conditions.

\section{IL-15 Availability Is a Limiting Factor for the Homeostatic Expansion of CIS-Deficient NK Cells in vivo}

Given that Cish-deficient NK cells appear to have a heightened sensitivity to IL-15 in vivo, we next considered whether this, and the lack of accumulation of NK cells may reflect a consequence of the Cish-deficient environment. To do this, we prepared mixed bone marrow (BM) chimeras. Peripheral NK cells from these mice remained at the same proportion as total donor lymphocytes anywhere from 6 to 24 weeks after reconstitution (Figure $\mathbf{3 A}$ ), and in addition, Cish-deficient NK cells maintained an elevated proportion of KLRG1 ${ }^{+}$NK cells (Supplemental Figure 3A). This was despite the increase in the total $\mathrm{KLRG}^{+}$population of $\mathrm{Cish}^{+/+} \mathrm{NK}$ cells in these chimeras when compared to steady-state, control mice. This suggests there is an increase in turnover rates of NK cells in BM transplant models, which may be caused by the initial total body irradiation (TBI) of the mice, which has shown to produce higher levels of soluble IL-15 and may impact the early stages of NK cell development (27). However, due to the outgrowth of $\mathrm{Cish}^{-/-}$ NK cells in vitro, this suggests the peripheral IL-15 concentration in both TBI and unconditioned $\mathrm{Cish}^{+/+}$and $\mathrm{Cish}^{-/-}$hosts is below the threshold to stimulate the IL-15 signaling pathway to induce Cish expression, thus unable to provide a survival and growth advantage to Cish deficient NK cells in vivo. To explore this further, we used mice that lack post-transcriptional control of IL-15 gene expression as hosts for mixed BM chimeras (referred to as IL-15Tg). IL-15Tg mice are able to translate and secrete an abundance of murine IL-15 protein, and intact mice display an expansion of NK cell numbers compared to wildtype controls (28). Surprisingly, even in this situation where IL-15 production is substantially increased, peripheral Cish-deficient NK cells again remained at the same proportion as total donor lymphocytes and could not outcompete Cish-sufficient NK cells (Figure 3B). This was again despite a total increase in KLRG1 expression on both subsets (Supplemental Figure 3B). In a co-culture, in vitro setting of IL-15 stimulation, it is apparent that Cish-deficient NK cells are hyper-proliferative and superior to Cish-sufficient NK cells. Since over-expression of IL-15 in vivo could not induce or replicate this competitive advantage, we next questioned whether the presence of IL-15 responsive lymphocytes (other than NK cells) could be causing the homeostatic maintenance of Cishdeficient NK cells.

Studies show the lack of NK and CD8 T cells in Rag2 ${ }^{-/-} \gamma_{\mathrm{c}}^{-/-}$ mice causes the ablation of homeostatic IL-15 sinks, creating an abundance of free soluble IL-15 in the periphery of these mice (29). To address whether the ablation of IL15 responsive cells (thus an increase in physiological IL-15) could overcome the homeostatic balance between $\mathrm{Cish}^{-1-}$ and $\mathrm{Cish}^{+/+} \mathrm{NK}$ cells in vivo, Cish ${ }^{-/-}\left(\right.$Ly5.1 $1^{-}$Ly5.2 $\left.{ }^{+}\right)$and Cish ${ }^{+/+}\left(\right.$Ly5.1 ${ }^{+}$Ly5.2 $\left.2^{+}\right)$NK cells were sorted and adoptively transferred into $\operatorname{Rag} 2^{-/-} \gamma_{\mathrm{c}}{ }^{-/-}$mice and their persistence and contribution to the NK cell compartment monitored over 16 weeks. Improved survival of $\mathrm{Cish}^{-/-} \mathrm{NK}$ cells was observed at all time-points and in all analyzed organs post transfer (Figure 3C and Supplemental Figure 3C). The outgrowth of Cish-deficient NK cells was accompanied by their sustained increase in the cell-cycle marker, Ki67 compared to WT NK cells (Supplemental Figure 3D). This suggests that the concentration of IL-15 in $R a g 2^{-/-} \gamma_{c}-/-$ mice is high enough to saturate the turnover rate of $\mathrm{NK}$ cells in vivo and confers a growth and survival advantage to Cish-deficient NK cells. While the concentration of soluble IL-15 in TBI mice may be increased at the time of NK cell development in mixed BM chimeras, these data suggest that other $\gamma$-chain expressing lymphocytes are still present and acting as homeostatic cytokine sinks, reducing the available IL-15 for NK cells during reconstitution. To determine whether other IL-15 responsive cells are the cause for the lack of expansion of Cish-deficient NK cells, we next generated mixed BM chimeras using Rag $2^{-/-} \gamma_{\mathrm{c}}{ }^{-/-}$mice as hosts. Despite the outgrowth of Cish-deficient NK cells when in an adoptive transfer setting, when $R a g 2^{-/-} \gamma_{\mathrm{c}}-/-$ mice were used as hosts in a chimeric setting, the competitive advantage of $\mathrm{Cish}^{-/}$NK cells was lost. There was also no competitive advantage given to the $\mathrm{Cish}^{-1-} \mathrm{CD}^{+} \mathrm{T}$ cells (Figure 3D). A previous study showed that depletion of CD8 $\mathrm{T}$ cells allowed more room for NK cells to expand, however they also showed that in WT mice that this CD8 depletion alone was not enough to encourage NK cell expansion (30). We hypothesized that the absence of CIS would enhance NK cell sensitivity to the unused IL-15 generated from the absence of CD8 T cells, thus breaking NK cell homeostasis. Mice were depleted of CD8 T cells using a CD8 depleting antibody, and loss of the CD8 $\mathrm{T}$ cell population was confirmed by FACS analysis (Supplemental Figure 3E). To our surprise, depletion of CD8s from reconstituted $\operatorname{Rag} 2^{-1-} \gamma_{\mathrm{c}}{ }^{-/-}$chimeric hosts did not alter the proportions of $\mathrm{Cish}^{+/+}$and $\mathrm{Cish}^{-/-} \mathrm{NK}$ cells from their initial 50/50 reconstitution (Figure 3E), suggesting that IL-15 concentrations were not restored to those in $\operatorname{Rag}^{-/-} \gamma_{\mathrm{c}}{ }^{-/-}$ mice. Similarly, CD8 depletion in intact $\mathrm{Cish}^{-/-}$mice did not alter NK cell percentage when compared to WT depletion (Supplemental Figure 3F). The implications from these data are two-fold; (1) CD8 T cells may not be the major IL-15 sink in vivo or (2) other $\gamma$-chain responsive lymphocytes are responsible for the regulation of NK cell numbers. In either situation, IL-15 availability appears to dictate NK cell expansion, and in steadystate situations there remains alternate regulatory mechanisms in place to maintain NK cell homeostasis.

\section{Loss of the Pro-apoptotic Protein, BIM, Does Not Alter the Homeostatic Expansion or Anti-tumor Function of Cish-Deficient NK Cells}

Previous studies show limiting levels of IL-15 are non-mitotic but are sufficient to maintain NK cell survival by reducing the level of pro-apoptotic protein BIM (encoded by Bcl2l11, referred to as Bim hereafter) $(31,32)$. Thus, we next sought to conditionally delete Bim in Cish-deficient mice using $\mathrm{Ncr}^{i \mathrm{Cre}}$ mice to gauge the impact of apoptosis on CIS-null NK cell

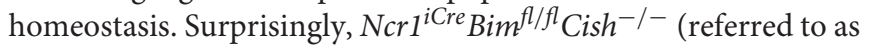
DKO hereafter) mice displayed similar numbers of NK cells to

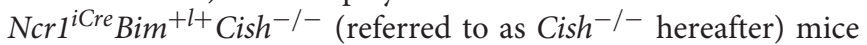


A

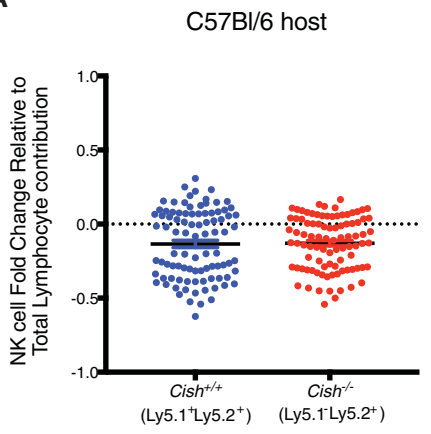

C
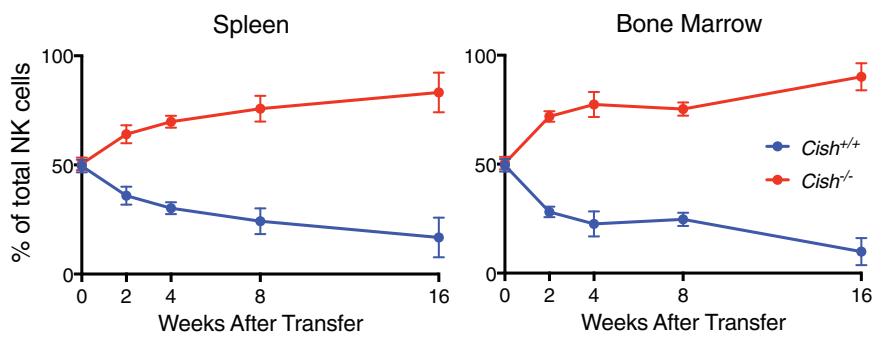

IL-15Tg host

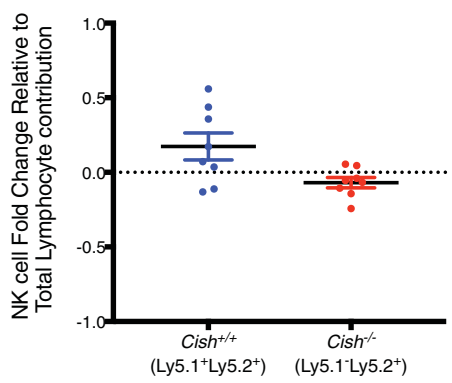

D

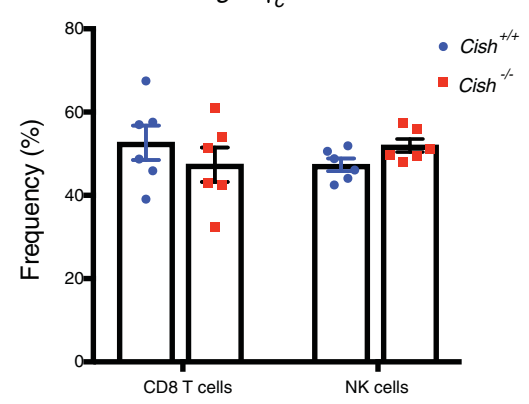

E

CD8 depletion of Rag2 ${ }^{--} \gamma_{c}^{-/}$hosts

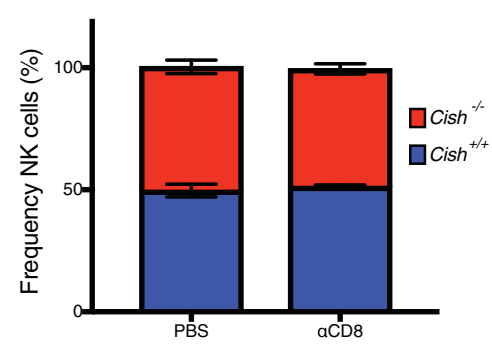

FIGURE 3 | IL-15 availability is a limiting factor for the homeostatic expansion of CIS-Deficient NK Cells in vivo. (A,B) Haematopoietic chimeras were generated by injecting irradiated host mice (C57BI/6 or IL-15Tg) with equal amounts of control Cish ${ }^{+/+}\left(\right.$Ly5. $1^{+}$Ly5.2 $2^{+}$) and Cish ${ }^{-1-}\left(\right.$ Ly5.2 $2^{+}$) bone marrow. After 6 weeks, NK1. $1^{+} \mathrm{NKp} 46^{+} \mathrm{CD} 49 \mathrm{~b}^{+}$cells were analyzed for expression of Ly5.1 and Ly5.2 to deduce donor origin). (C) Adoptive transfer models were generated by injecting

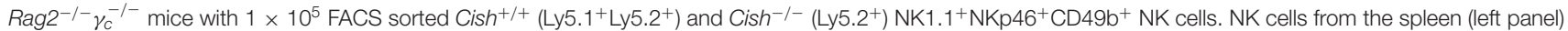
and BM (right panel) were analyzed for expression of Ly5.1 and Ly5.2 to deduce donor origin at indicated time points. (D) Haematopoietic chimeras were generated using irradiated Rag2 ${ }^{-1-} \gamma_{c}{ }^{-1-}$ mice as hosts and NK and T cells from each donor were assessed as described earlier. (E) Reconstituted Rag2 $2^{-/-} \gamma_{c}{ }^{-1-}$ mice were injected i.p. once per week for 4 weeks with anti-CD8 to deplete T cells, and splenic NK cells were analyzed for expression of Ly5.1 and Ly5.2 to deduce donor origin in control (PBS treated) or $\alpha$ CD8 treated reconstituted Rag2 ${ }^{-1-} \gamma_{c}^{-1-}$ hosts. (A,B, $n \geq 4$ biological replicates mean \pm s.e.m.; C, mean \pm s.e.m. of $n=3$ biological replicates at each timepoint; $\mathbf{D}, \mathbf{E}, n=6$ biological replicates mean \pm s.e.m.).

upon phenotypic analysis (Figure 4A). To understand this in more detail, we cultured DKO and $\mathrm{Cish}^{-/-} \mathrm{NK}$ cells in a nonmitogenic concentration of IL-15 and found DKO NK cells appear to survive better under these conditions (Figure 4B). These data suggest that while the pro-apoptotic protein, BIM, may act as an alternative checkpoint in the absence of CIS to ensure the death of rapidly cycling NK cells and maintain homeostatic numbers, in the absence of BIM, Cish ${ }^{-1-}$ mice continue to maintain homeostatic NK cell numbers in vivo.

There are a number of factors that suppress $\mathrm{NK}$ cells in the tumor microenvironment (TME), either directly or indirectly, which can affect NK cell proliferation, effector functions and infiltration [reviewed by (33)]. In the TME, it must be considered that while IL-15 may be upregulated due to the chronic inflammatory nature of tumor formation, other suppressive mechanisms can override robust IL-15 signaling in NK cells under these circumstances. Previous studies have shown Bim $^{-/-}$NK cells can survive IL-15 withdrawal significantly better than WT NK cells and that this is sustained over time (31). This also remains true for DKO NK cells in vitro. Thus, we next wanted to look at whether the survival of DKO NK cells could further improve the anti-tumor response 
A

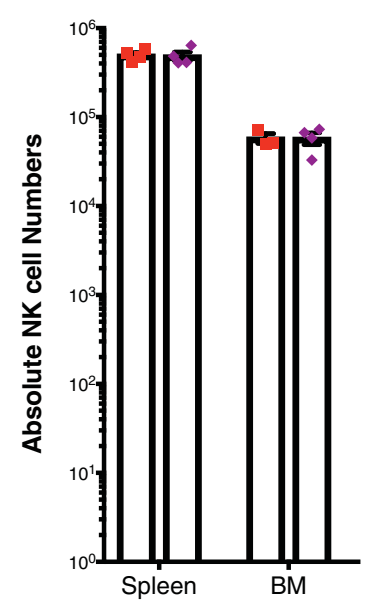

C

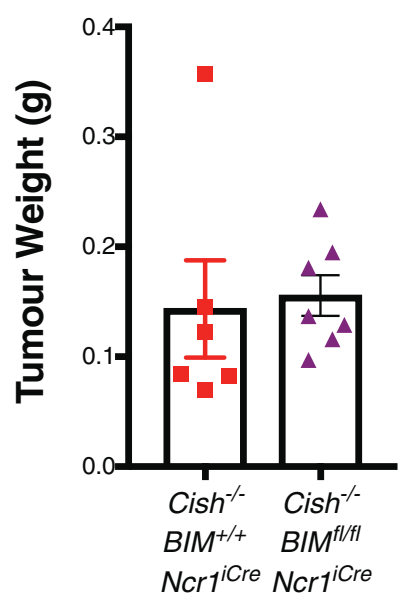

B

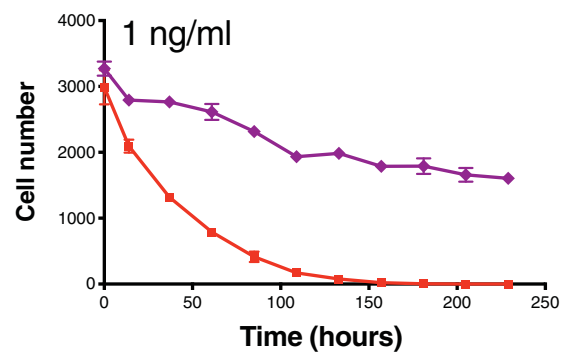

D

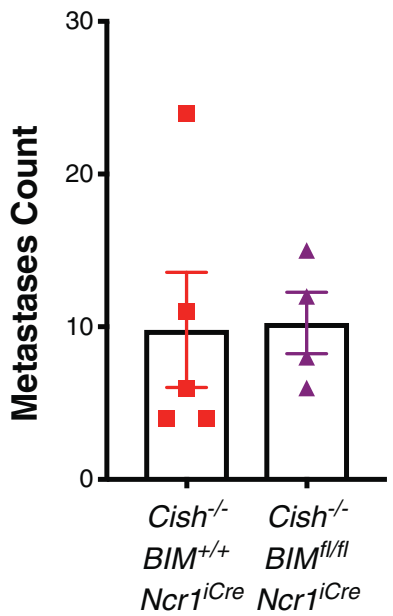

FIGURE 4 | Loss of the pro-apoptotic protein, BIM, does not alter the homeostatic expansion or anti-tumor function of Cish-deficient NK cells. (A) Absolute numbers

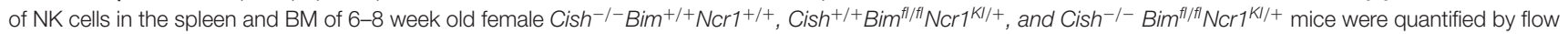
cytometric analysis. (B) Purified and CTV-labeled Cish ${ }^{-1-}$ Bim $^{+/+} \mathrm{Ncr} 1^{i C r e}$, and Cish $^{-1-}$ Bim $^{f / f l} \mathrm{Ncr}^{\text {iCre }}$ NK cells were seeded at $1 \times 10^{4}$ cells/well into round wells containing $1 \mathrm{ng} / \mathrm{ml} \mathrm{IL}-15$. Cells were incubated at $37^{\circ} \mathrm{C}$ in a humidified environment containing $5 \% \mathrm{CO}_{2}$ for $240 \mathrm{~h}$. Total cell numbers over time are presented. (C) $1 \times$ $10^{6}$ SM1-LWT1 tumor cells were injected s.c. into flanks of Cish ${ }^{-1-}$ Bim $^{+/+} \mathrm{Ncr} 1^{\text {iCre }}$ and Cish ${ }^{-/-}$Bim $^{\text {fl/fl }} \mathrm{Ncr}^{\text {iCre }}$ mice. After 2 weeks, tumors were excised and volume

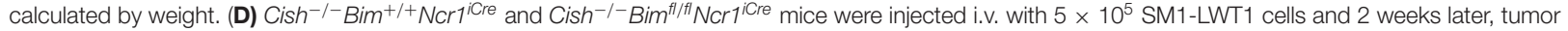
metastases were enumerated. (A, $n=4$ biological replicates mean \pm s.e.m.; B, mean \pm s.e.m. of $n=3$ biological replicates at each timepoint; $\mathbf{C}, \mathbf{D}, n \geq 4$ biological replicates of one experiment representative of two independent experiments with similar results, mean \pm s.e.m.).

of $\mathrm{Cish}^{-/-}$mice. The SM1-LWT1 cell line is a metastatic melanoma line and sensitive to NK cell killing (34). We showed in both a sub-cutaneous and i.v. model of SM1-LWT1 that Cish-deficient and DKO were similar in their rejection of tumors (Figures 4C,D). Whilst we have previously shown that Cish-deficient NK cells are superior at the clearance of tumors, there was no definitive evidence as to what caused this enhanced anti-tumor function. That Cish-deficient NK cells still show significant death in low concentrations of IL15 combined with the similar anti-tumor response observed in DKO mice suggests that increased survival is not a factor contributing to the anti-tumor function of Cish-deficient NK cells in vivo.

\section{CIS Regulates Global Gene Expression in NK Cells}

Since the absence of BIM did not confer a survival or homeostatic advantage to Cish-deficient NK cells in vivo, we next used RNA sequencing to identify aberrant candidate pathways or genes intrinsic to $\mathrm{Cish}^{-/-} \mathrm{NK}$ cells that may be responsible for regulating their homeostasis in vivo. We performed 75bp single-ended RNA sequencing on $\mathrm{Cish}^{+/+}$and $\mathrm{Cish}^{-/-}$ 
NK cells purified directly from the spleen. Surprisingly, more than 3,000 differentially expressed genes were detected in $\mathrm{Cish}^{-1-}$ NK cells. Of these, more than half of the genes expressed at fold-change of 1.5 or higher, and the top $50 \mathrm{DE}$ genes were downregulated, suggesting that a number of active pathways are dampened in Cish ${ }^{-1-}$ NK cells in an attempt to counteract the increased turnover and prevent accumulation (Figure 5A). Gene set analysis was indicative of this, with various metabolism pathways downregulated (Figure 5B). From the gene set analysis, it was quite evident the genes that fall within pathways initiated through IL-15 signaling (MAPK, JAK/STAT, mTOR) are upregulated, confirming that Cish-deficient NK cells are hypersensitive to IL-15 in vivo. However, genes involved downstream of these IL-15 initiated pathways (p53 signaling, DNA replication, and metabolism) are significantly downregulated. This suggests the secondary checkpoints that prevent accumulation are downstream of gene transcription directly initiated by IL-15. Notably, the purine and pyrimidine metabolism pathways were significantly downregulated, along with DNA replication (Figure 5B). In T cells, purine/pyrimidine metabolic processes regulate cell cycle progression and survival, and abrogation of these pathways results in apoptosis (35). The significant downregulation of these pathways in Cish-deficient NK cells in vivo may help explain inhibitory pathways wired into NK cells to increase apoptosis and prevent accumulation. In addition, a number of genes involved in the apoptosis pathways were upregulated, validating our EdU incorporation results.

Although we observed cyclic abnormalities in Cish-deficient NK cells in vivo, gene set enrichment analysis showed genes required for NK cell cytotoxicity, FcR $\gamma$ signaling and chemokine signaling were upregulated. Genes important for activation and degranulation such as Ifng, Gzma, Gzmb, Prfl, and Serpinb9 were upregulated, along with genes downstream of NKG2D and NKp46 activating receptors such as Hcst, Tyrobp, Zap70, and Syk (Figure 5C). Previously published CHIP-seq data showed that certain STAT5 target genes (such as Ifng) are already bound to STAT5 under tonic IL-15 conditions (in vivo), and that stimulation with IL-15 increases the amplitude of the gene and number of new binding sites (36). Since tonic levels of IL-15 are similar in Cish-deficient and wildtype mice, the increase in STAT5 target gene RNA suggests that Cish-deficient NK cells are more sensitive to tonic IL-15 in vivo. Increased sensitivity to IL-15 and subsequent enhanced expression of STAT5 target genes such as Ifng and Gzmb are suggestive of improved activation. As we have seen previously, the absence of CIS confers superior cytotoxicity and subsequent anti-tumor responses. Our data thus far argues that the mechanism behind this is not attributed to tumor infiltration or survival within the tumor microenvironment. Gene set analysis of Cish-deficient NK cells shows significant upregulation of pathways and genes associated with NK cell cytotoxicity and activation, and heavily suggests that enhanced anti-tumor function is a direct result of improved cytotoxicity. Interestingly, this is not confined to one singular signaling pathway, but appears to span across a broad range of activating receptors and cytotoxic pathways suggesting that under homeostatic conditions CIS regulates NK cell activation.

\section{CIS Lowers the Activation Threshold in NK Cells and Increases Anti-tumor Function on a per Cell Basis}

The enrichment of genes downstream of activating receptors such as NK1.1, NKp46, and NKG2D suggests a more activated state of Cish-deficient NK cells in vivo. Thus, we next measured whether there were any alterations in activating receptor expression on the surface of $\mathrm{Cish}^{-/-}$and $\mathrm{Cish}^{+/+} \mathrm{NK}$ cells and found no differences (Figure 6A). Our prior studies showed germline deletion of CIS in NK cells manifested in superior IFN $\gamma$ production when co-stimulated with IL-15 and activating receptors NK1.1 or NKp46 (9). Intriguingly, when ex-vivo, germline $\mathrm{Cish}^{-1-}$ NK cells were stimulated through NK1.1 and NKp46 in the absence of IL-15, we also observed increases in IFN $\gamma$ production and degranulation compared to $\mathrm{Cish}^{+/+}$ NK cells (Figure 6B). The functional output from non-specific stimulation (phorbol myristate acetate (PMA) in combination with the calcium ionophore, ionomycin, or IL-12 and IL18 co-stimulation) was also measured to quantify maximal effector function and interestingly, both IFN $\gamma$ production and degranulation were equally represented between both genotypes (Supplemental Figure 3G). This suggests that Cish-deficient NK cells do not have excess cytokine and degranulation capabilities, but rather, can degranulate and produce cytokine more rapidly from their resting state and suggests that the in vivo sensitivity of NK cells to IL-15 establishes a reduced activation threshold. Together with the upregulation of activating genes, it appears that increased sensitivity to IL-15 in vivo results in NK cells being "pre-primed" or reducing their activation threshold and that any increases in $\mathrm{Cish}^{-/-} \mathrm{NK}$ cell activation is not an effect of receptor abundance or intensity, but rather downstream effects of the activating receptors.

To evaluate this pre-primed phenotype in vivo, we first assessed the differences in cytotoxic capacity between Cish-deficient and WT NK cells against an immunogenic methylcholanthrene (MCA)-induced fibrosarcoma cell line (MCA1956). We found that when challenged in vitro, $\mathrm{Cish}^{-/-}$ NK cells were more efficient at lysing MCA tumor cells at a range of effector:target (E:T) cell ratios (Figure 6C). In vivo, $100 \% \mathrm{Cish}^{-/-}, 100 \% \mathrm{Cish}^{+/+}$or 50/50 competitive chimeras were challenged with the MCA1956 cell line, and tumor burden was decreased in both the Cish-deficient chimeras as well as the competitive chimeras (Figure 6D). When intratumoural NK cell proportions were examined within the 50/50 $\left(\mathrm{Cish}^{-/-} / \mathrm{Cish}^{+/+}\right)$competitive chimeras, in line with our previous reconstitution data from the $\mathrm{BM}$ and spleen, the NK cell proportions within the tumor and in peripheral organs remained at 1:1 (Figure 6E). Additionally, in $100 \%$ chimeras, despite the decrease in tumor volume, the absolute number of $\mathrm{Cish}^{-/-} \mathrm{NK}$ cells per gram of tumor was not increased compared to Cish ${ }^{+/+}$NK cells (Figure 6F). Together with our previous data that showed in the absence of BIM, NK cell anti-tumor function does not appear to benefit from increased survival, these data suggest that the failure to outcompete WT NK cells in the tumor setting does not compromise the anti-tumor function of Cish-deficient NK cells 
A

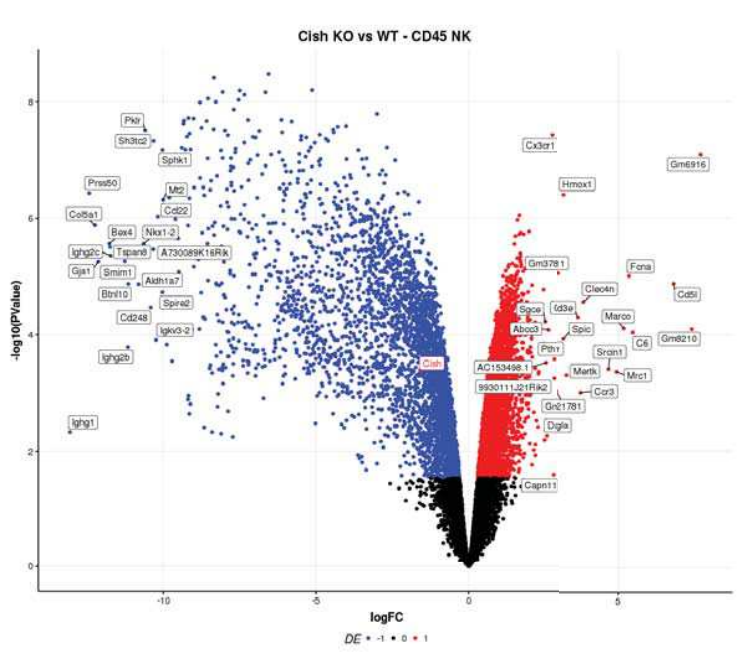

B

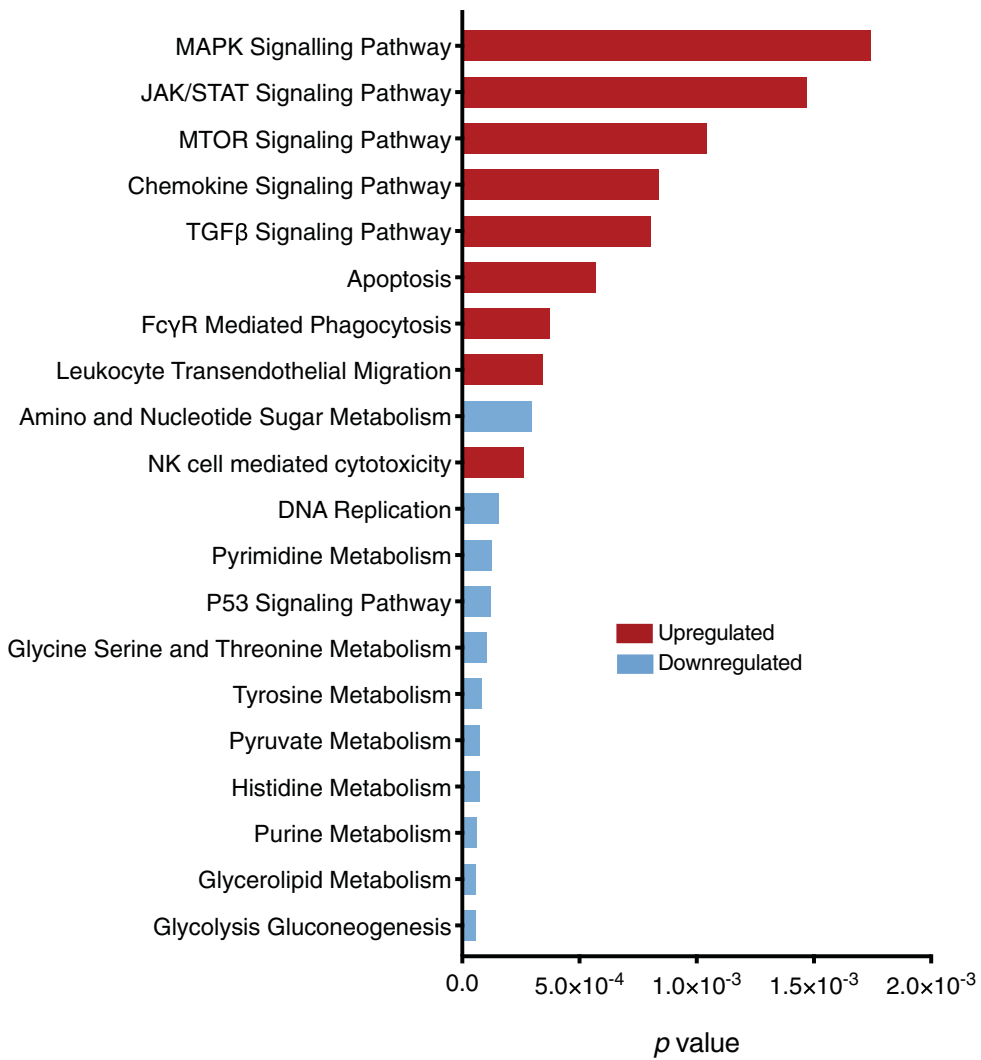

C

\section{NK Cell mediated Cytotoxicity}

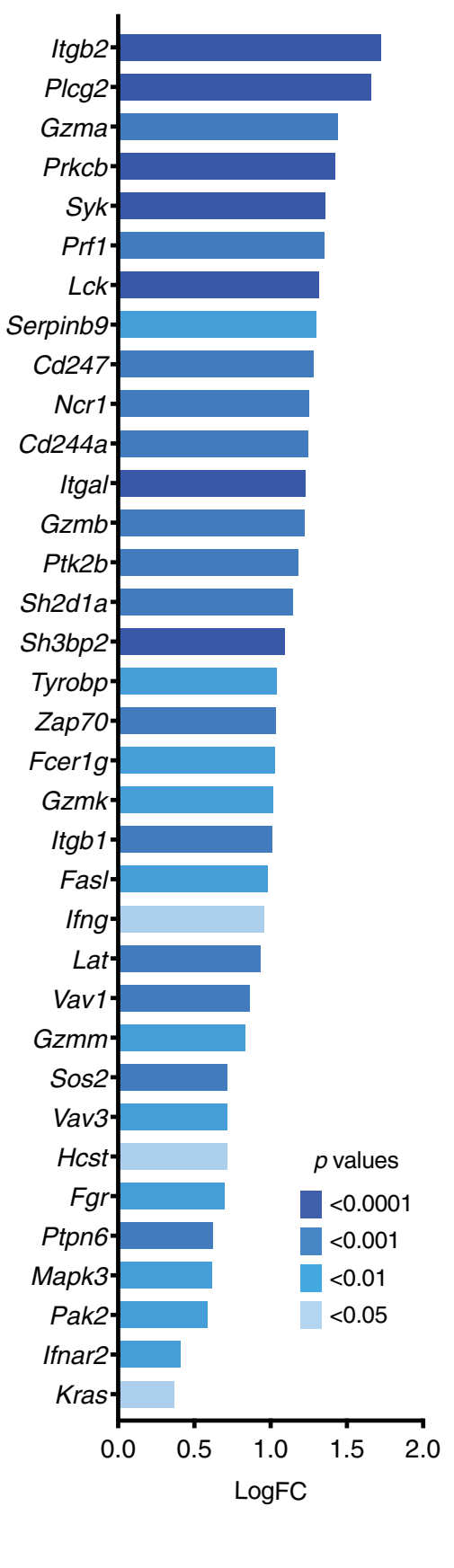

FIGURE 5 | CIS regulates global gene expression in NK cells. Gene expression profiles of sorted NK cells were generated using RNA-sequencing. (A) A volcano plot of the top 50 significant $(p<0.05)$ differentially expressed genes with a fold change above +1 or below -1 was generated. $y$-axis is the negative log-p-value and $\mathrm{x}$-axis is the log-fold-change of the corresponding gene in Cish ${ }^{-/-}$vs Cish ${ }^{+/+}$comparison. Gene expression: blue $=$downregulated, red $=$upregulated. (B) A list of genes was generated using a $p$-value of $<0.05$ and a cutoff of $\log (F C)=1.5$. The resulting genes were run through $K E G G$ pathway analysis and 20 of the top enriched KEGG pathways with a $p$-value of $<0.01$ were plotted. The height of the bar indicates the significance ( $p$-value). Pathways: blue $=$ downregulated in $\mathrm{Cish}^{-/-}$, red = upregulated in Cish ${ }^{-1-}$. (C) An upregulated list of genes associated with NK cell cytotoxicity (from KEGG pathway analysis) was generated with a $p$-value cut-off of $<0.05$. $x$-axis is the $\log (\mathrm{FC})$ of each gene and $p$-values are indicated by color. 
A

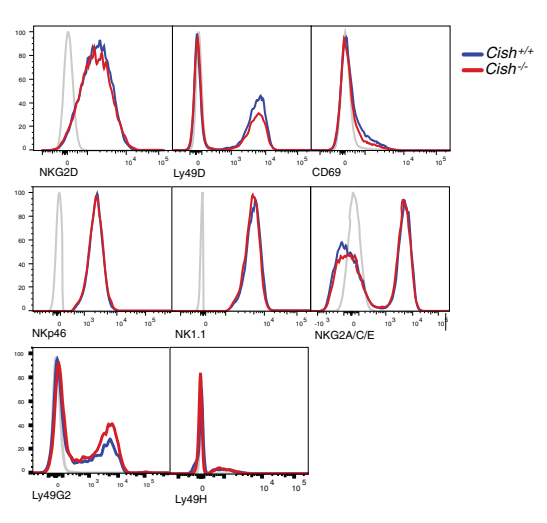

B
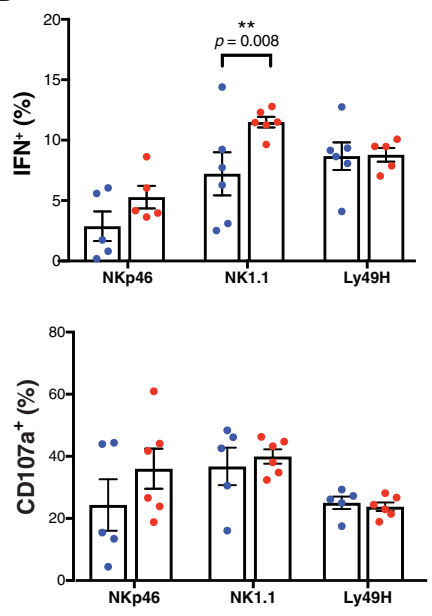

D

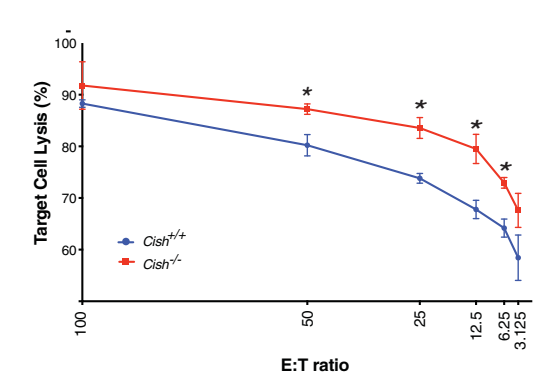

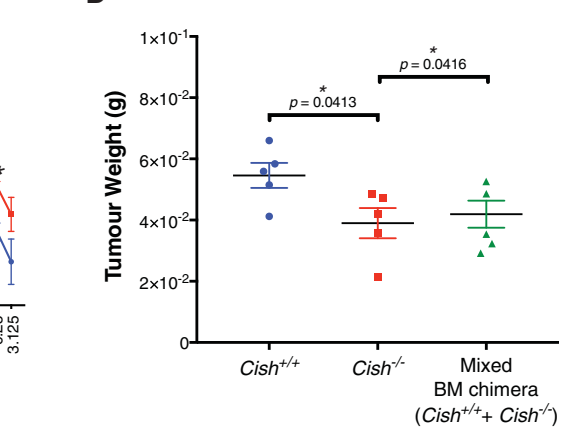

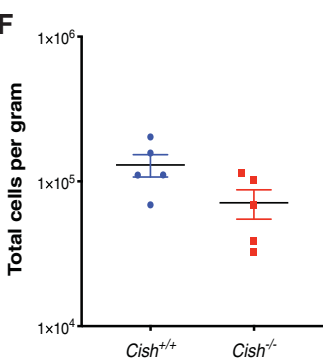

$\mathrm{Cish}^{+/ 4} \quad \mathrm{Cish}^{\mathrm{*}}$
E

MCA-challenged mice

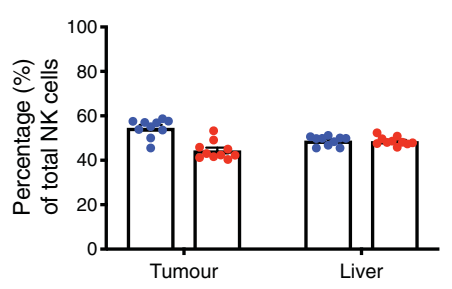

FIGURE 6 | CIS lowers the activation threshold in NK cells and increases anti-tumor function on a per cell basis. (A) Splenic NK cells from Cish ${ }^{+/+}$and Cish ${ }^{-/-}$mice were phenotypically analyzed by flow cytometry for activating receptor expression. Representative histograms of receptor expression are shown. (B) Total splenic cells were harvested from 6 to 8 week-old Cish ${ }^{+/+}$and Cish ${ }^{-/-}$mice and depleted of red blood cells. Cells were cross-linked with anti-NK1.1, anti-NKp46, and anti-Ly49H antibodies for $4 \mathrm{~h}$ in the absence of IL-15. Flow cytometric analysis of NK cells and their IFN- $\gamma$ production and CD107a (LAMP-1) expression was assessed after $4 \mathrm{~h}$. ${ }^{* *} p<0.01$ (unpaired Student's $t$-test). (C) Splenic NK cells were purified from non-tumor bearing Cish ${ }^{+/+}$and Cish ${ }^{-/-}$mice and cultured with MCA targets at the indicated effector:target (E:T) ratios. Target cell lysis was quantified by detecting the release of a fluorescent cytoplasmic dye into the culture media after $4 \mathrm{~h} .{ }^{\star} p<0.05$ (unpaired Student's $t$-test). (D) Female 6-8 week-old reconstituted Cish ${ }^{+/+}$, Cish ${ }^{-/-}$and mixed Cish ${ }^{+/+}$and Cish ${ }^{-/-}$BM chimeras were subcutaneously inoculated with $1 \times 10^{5}$ MCA cells. Tumors were excised after 14 days and weighed. ${ }^{*} p<0.05$ (unpaired Student's $t$-test). (E) Tumors and livers from mixed Cish ${ }^{+/+}$and Cish ${ }^{-/-}$ BM chimeras were excised 14 days after tumor inoculation and NK cell infiltrate was measured using flow cytometric analysis of Ly5.1 and Ly5.2 to deduce NK cell donor origin. (F) Tumors from reconstituted Cish ${ }^{+/+}$and Cish ${ }^{-1-}$ mice were analyzed using flow cytometry and total NK numbers enumerated. $(\mathbf{A}$, representative plots from $n=3$ biological replicates; B, mean \pm s.e.m. of $n=3$ biological replicates from one experiment representative of three independent experiments with similar results. $\mathbf{C}$, mean \pm s.e.m. of $n=3$ biological replicates at each E:T ratio timepoint; D-F, mean \pm s.e.m of $n \geq 5$ biological replicates of one experiment representative of two independent experiments with similar results).

and highlight their increased cytotoxicity on a per cell basis. Thus, the upregulation of cytotoxicity and activating receptor genes in the absence of CIS enhance the effector capabilities of NK cells.

\section{DISCUSSION}

NK cell homeostasis is a tightly controlled event that encompasses a number of complex processes and signaling 
events. IL-15 is the crucial factor inducing NK cell proliferation, survival, and differentiation in vivo $(37,38)$. The present data identifies a role for CIS in murine NK cell homeostasis and suggests a mechanism for the enhanced anti-metastatic function previously published in Cish-deficient mice (9). Whilst tonic levels of IL-15 were initially thought to render the role of CIS redundant under homeostatic conditions, Cish-deficient NK cells are terminally mature, show enhanced cycling (Ki67 expression, EdU uptake) and turnover (loss of Edu), arguing in favor of a physiological role for CIS in vivo. However, additional safety mechanisms or checkpoints appear to be in place to prevent NK cell accumulation in the absence of this potent negative-regulator of IL-15 receptor signaling.

Alterations in NK cell numbers during health and disease are rarely observed in mice or humans. An exception being the large transient increase in NK cell numbers during murine or human cytomegalovirus (CMV) infection which returns to baseline 2 weeks post-infection and is driven by a viral peptide, not IL-15 $(39,40)$. NK cell numbers in the periphery of mice lacking genes important for NK cell survival, proliferation, differentiation, or function show very little change in NK cell numbers. In mice where the loss of a gene can dramatically alter NK cell biology in vitro, NK cell numbers in vivo are not altered. Cish-deficient mice (improved IL-15-dependent survival/proliferation of NK cells) and Bcl2l11-deficient mice [whereby Bim deficiency in NK cells results in a dramatic survival advantage in the absence of IL-15 (31)] are examples of this as both of these models harbor NK cell frequencies equal to that of littermate controls under homeostatic conditions. This is in vast contrast to similar genetic alterations in $\mathrm{T}$ and $\mathrm{B}$ cells, which result in their accumulation over time leading to immunopathology and autoimmunity $(41,42)$.

In humans, this tight regulation of NK cells is conserved, evidenced by the rarity of lymphoproliferative disorders of NK cell origin, which account for less than 5\% of all lymphoid neoplasms (43). Perhaps even more interesting, is that in the rare cases where NK cell numbers become dysregulated, such as aggressive NK cell leukemia (ANKCL), the prognosis is extremely poor, with a mean survival rate of 2-3 months after diagnosis (44). This in itself may be evidence for why NK cell numbers have a number of safeguards or checkpoints in place when it comes to their regulation in vivo. Surprisingly, removing Bim-dependent intrinsic apoptosis from NK cells that are hyper-responsive to IL$15\left(\mathrm{Ncrl}^{i \mathrm{Cre}} \mathrm{Bim}^{\mathrm{fl} / \mathrm{fl}} \mathrm{Cish}^{-/-}\right)$did not result in an accumulation of NK cells in vivo. It is possible, that alternate apoptotic regulators such as Noxa and Puma are also upregulated in Cish ${ }^{-/-}$NK cells, and that this can compensate for a loss of Bim.

In an attempt to understand how CIS regulates essential NK cell functions, we were able to demonstrate that NK cell increased anti-tumor efficacy in the absence of CIS is not due to NK cell accumulation, increased survival or infiltration at the tumor site. However, our gene set enrichment analysis (GSEA) shows upregulation of signaling pathways essential for NK cell cytotoxicity. This was accompanied by increases in IFN $\gamma$ production and CD107a expression upon activating receptor stimulation in vitro.

The regulation of NK cell responses to infectious or tumor cells is regulated by the balance between activating and inhibitory signals [summarized by (45)]. This implies the existence of an activation threshold, where a certain level of activation must outweigh the inhibitory signals in order to elicit a functional response (46). This study suggests CIS maintains an activation threshold, and that by inhibiting CIS, increased sensitivity to IL-15 is generated and lowers the activation threshold to elicit a functional response. The absence of exogenous IL-15 during the $4 \mathrm{~h}$ stimulation assays ensures no induction of CIS, thus it is likely the levels of IL-15 in vivo are responsible for the pre-primed response of Cish-deficient NK cells compared to WT.

Additionally, it was showed in the early 1980s that a proportion of NK cells are capable of killing multiple targets in succession (47). Additionally, recent reports suggest that IL-15 can improve the killing frequency of NK cells by augmenting the number of targets an NK cell can kill before they become exhausted (48). This study shows Cish-deficient NK cells have the ability to lyse more target cells than WT NK cells both in vitro and in vivo and suggests that CIS regulates, at least in part, the killing frequency of NK cells.

How CIS imposes restrictions on the number of targets an NK cell can kill may occur in a number of ways. Cishdeficient NK cells show differential upregulated expression of the gene Serpinb9. Serpinb9 prevents cell death by inactivating proteases such as granzyme B that are released into the host cell interior (49). This suggests a mechanism by which Cishdeficient NK cells harbor an increased killing frequency when they encounter tumor metastases in vivo. More studies are required to understand the precise mechanisms behind these initial observations, but it suggests that CIS is an early inhibiting molecule of several signaling pathways that regulate NK cell activation thresholds.

In conclusion, targeting CIS improves NK cell ex-vivo proliferation, but also demonstrates no homeostatic or developmental differences or abnormal accumulation when CIS is deleted in vivo. These results emphasize the safety of targeting CIS, promoting improved anti-tumor functions without any apparent secondary or off-target effects. Importantly, we also highlight the targeted deletion of CIS as a means of lowering the NK cell activation threshold, in turn enhancing anti-tumor function using standard methods of stimulation. Thus, in this study we confirmed and further characterized the inhibition of CIS as a method of unleashing the NK cell anti-tumor response. We propose that releasing NK cell inhibition by targeting CIS is a novel strategy to improve NK cell anti-tumor properties and recommend their use in clinical immunotherapy and adoptive transfer approaches.

\section{METHODS}

\section{Mice}

$\mathrm{Cish}^{-/-}$were generously provided by J. Ihle and E. Parganas (St. Jude Children's Research Hospital) and were maintained on a C57BL/6 background. Cish ${ }^{+/+}$refers to $\mathrm{C} 57 \mathrm{BL} / 6$ wildtype control mice. Age and sex matched mice were used and cohort size was dictated by previous experience using these tumor models. Mice were bred and maintained under specific pathogenfree conditions at The Walter and Eliza Hall Institute. Animal 
experiments followed the National Health and Medical Research Council (NHMRC) Code of Practice for the Care and Use of Animals for Scientific Purposes guidelines and were approved by the Walter and Eliza Hall Institute Animal Ethics Committee.

\section{Genotyping}

$B c l 2 l 11^{f l / f l}$ genotyping was performed using the following PCR primers: Bim9, 5'-GACAAGGTGGACAATTGCAG-3'; PB173, 5'-AACCAACTGTACCTTGGCTATA-3'; with expected band sizes at $620 \mathrm{bp}$ for WT alleles and $920 \mathrm{bp}$ for floxed allele. $\mathrm{Ncrl}^{i \mathrm{Cre} /+}$ genotyping was performed using the following PCR primers: iCRE Fwd, 5'-GGAACTGAAGGC AACTCCTG-3'; iCRE Fwd KI, 5'-GTCCATCCCTGAAATC ATGC-3'; Rev WT:-5' TTCCCGGCAACATAAAATAAA-3' with expected bands sizes at $300 \mathrm{bp}$ for WT allele and $376 \mathrm{bp}$ for KI allele.

\section{Generation of BM Chimeras}

All BM chimeras were generated using the same method. Host mice were lethally irradiated $(2 \times 550$ rads $)$ and reconstituted with either $7 \times 10^{6}$ donor BM cells for $100 \%$ chimeras or 3.5 $\times 10^{6}$ control donor $\mathrm{BM}$ and $3.5 \times 10^{6}$ experimental donor BM. Mice were kept on neomycin-treated water for 3 weeks, and reconstitution of $\mathrm{BM}$ compartment was monitored from 6 weeks onwards.

\section{NK Cell Cytotoxicity Assays}

Standard $4 \mathrm{~h}$ cytotoxicity assays were completed as described elsewhere (50). Briefly, splenic NK cells were isolated and suspended in NK cell medium (phenol-red free RPMI 1640 containing $10 \%$ FCS, non-essential amino acids, L glutamine and sodium pyruvate, all from Gibco). The indicated target cells were labeled with $15 \mu \mathrm{M}$ Calcein-AM (Life Technologies) for $30 \mathrm{~min}$ at $37^{\circ} \mathrm{C}$, washed twice and suspended in NK cell medium. Effector and target cells were combined at the indicated ratios in triplicate wells of a round-bottom 96 well plate and incubated at $37^{\circ} \mathrm{C} / 5 \%$ $\mathrm{CO}_{2}$ for $4 \mathrm{~h}$. Calcein release was quantified by transferring $100 \mu \mathrm{L}$ of cell-free supernatant to opaque 96 well plates and measuring fluorescent emission at the appropriate wave-length (excitation filter: $485 \pm 9 \mathrm{~nm}$; cutoff: $515 \mathrm{~nm}$; emission: $525 \pm 15 \mathrm{~nm}$ ) using the EnVision Robot Plate Reader.

\section{Flow Cytometry and Cell Sorting}

Single-cell suspensions were stained with the appropriate monoclonal antibody in PBS containing 2\% FCS. When necessary, intracellular staining was performed by use of the FoxP3/Transcription Factor Staining Buffer Set (eBioscience) according to the manufacturer's instructions. Fortessa, FACS Verse, and ARIAIII (BD Biosciences) were used for cell sorting and analysis. Antibodies specific for NK1.1 (PK136; 1:100), CD19 (1D3; 1:400), CD3 (17A2; 1:400 or REA641; Miltenyi Biotec; 1:150); CD122 (TM- $\beta 1 ; 1: 200)$, NKp46 (29A1.4; 1:100), KLRG1 (2F1; 1:200), CD27 (SB/199; 1:200), CD11b (M1/70; 1:200), IL-7R (A7R34; eBioscience; 1:200) CD49b (DX5; 1:100), CD49a (Ha31/8; 1:200) Ly49H (3D10; 1:200) Ly49D (4E5; 1:200), NKG2D (C4; 1:200), NKG2A/C/E (20d5; 1:200), Ly49C/I (5e6; $1: 100)$, CD107a (104B; 1:100), and IFN- $\gamma$ (XMG1.2; 1:100),
DNAM-1 (10E5; 1:200); Ki-67 (AF488; 1:50) were from BD Pharmingen unless stated otherwise.

\section{Cell Counts}

123 count eBeads (BD Bioscience) beads were added to single cell suspensions prior to flow cytometry. Cell numbers were enumerated according to manufacturers instructions.

\section{Enumeration of Apoptotic Cells}

The enumeration of apoptotic cells was performed using the CellEvent ${ }^{\mathrm{TM}}$ Caspase-3/7 Green Flow Cytometry Assay Kit (catalog \#: C10427; Thermo Fisher Scientific) following manufacturer's instructions.

\section{EdU NK Cell Turnover Assay}

EdU was prepared as per manufacturer's instructions, and either $200 \mu \mathrm{g} / 200 \mu \mathrm{l} /$ mouse or vehicle (DMSO) was injected i.p. on Day 0 and Day 2. Mice were then sacrificed at different timepoints. At each timepoint, spleen and BM from $1 \times$ femur were harvested from each mouse and made up as single cell suspension. Organs were lysed with RBC lysis buffer and stained for surface markers. EdU detection was followed exactly as per manufacturer's instructions (catalog \#: C10632; Thermo Fisher Scientific) and analyzed on the FACS Verse.

\section{Total Cell Number and Mean Division Number Determination}

The precursor cohort method used to determine mean division numbers has been published (51). Plotting the mean division number (MDN) of cell populations against time enables estimates and comparisons of division rates. MDN is the average number of divisions the initial cohort has undergone, where total cohort number describes the number of founding cells initially present within a population. To determine the MDN, each generation number $i$ is multiplied by the fraction of the cohort that had undergone $i$ divisions, and these values are summed for each generation:

$$
\text { Mean division number }=\sum\left(i \times \frac{\text { cohort number }}{i}\right)
$$

where $\mathrm{i}$ is division number.

\section{Plate Bound Assay}

For the measurement of IFN- $\gamma$ production, splenocytes were activated in 96 well plates with hIL-15 (50 ng/ml), IL-12 (10 ng/ml, Peprotech), IL-18 (100 ng/ml), PMA (10 ng/ml), Ionomycin $(1 \mu \mathrm{g} / \mathrm{ml})$, or coated with anti-NK1.1 $(25 \mu \mathrm{g} / \mathrm{ml}$, BioLegend), anti-NKp46 (10 $\mathrm{g} / \mathrm{ml}$, BioLegend), or $\mathrm{LY} 49 \mathrm{H}$ $(10 \mu \mathrm{g} / \mathrm{ml}$, BioLegend). Cells were incubated with monensin and brefeldin A (BD GolgiPlug and GolgiStop) in complete medium for $4 \mathrm{~h}$ at $37^{\circ} \mathrm{C}$. The cells were subjected to surface staining and intracellular staining was performed by use of the FoxP3/Transcription Factor Staining Buffer Set (eBioscience).

\section{Tumor Cell Lines}

MCA1956 fibrosarcoma cell line was derived from a B6.WT female mouse (gift of Robert Schreiber) and the SM1-LWT1 
melanoma cell line was generously provided by Mark Smyth. Both MCA1956 and SM1-LWT1 melanoma cell lines were cultured in DMEM media containing 10\% FCS (GE Healthcare Life Sciences), $100 \mathrm{U} / \mathrm{mL}$ penicillin (Sigma), $100 \mathrm{mg} / \mathrm{mL}$ streptomycin (sigma), $1 \mathrm{mM}$ glutamax (Gibco) and sodium pyruvate (Gibco).

\section{Experimental Tumor Metastasis}

Single-cell suspensions of $5 \times 10^{5}$ MCA1956 cells were injected s.c. into the tail vein of the indicated strains of mice or s.c. Mice were sacrificed and lungs and/or tumors were harvested on day 14. Tumor weights were recorded and lungs were injected with Indian Ink, washed twice in PBS and fixed in Feketes solution overnight to count metastases.

\section{Sample Preparation, RNA Sequencing, and Bioinformatics Analysis}

RNA isolation from sorted ex vivo NK cells was extracted using the RNeasy Plus Mini Kit (\#74134, QIAGEN, Hilden, Germany), according to the manufacturer's instructions. Purified RNA was measured using an Agilent 2200 TapeStation System (Agilent) with High Sensitivity (HS) RNA ScreenTapes (\#5067-5579, Agilent). Next-generation sequencing libraries were generated using 100 ng RNA from samples with distinct $18 \mathrm{~S}$ and $28 \mathrm{~S}$ peaks and RNA Integrity Number values $\geq 9$, using the NEBNext Ultra II Directional RNA Library Prep Kit for Illumina (\#E7760L, New England Biolabs) according to the manufacturer's instructions. Approximately 20 million reads per sample were obtained by pooling RNA libraries and performing single-end 75 bp sequencing. Sequencing was performed in the Genomics Laboratory at the Walter and Eliza Hall Institute on a NextSeq 500 next-generation sequencer (Illumina). Single-End reads 3576 bp in length corresponding to $\mathrm{Cish}^{-/-}$and WT $\mathrm{Cish}^{+/+}$ NK cells (three biological replicates per group) were quality checked using fastqc (52). Low quality bases and TrueSeq Adapters were trimmed using trimmomatic (53). Reads were aligned to $10 \mathrm{~mm}$ using STAR (54). The aligned reads were summarized at the gene-level using featureCounts (55). Genes were filtered from downstream analysis if they failed to achieve a CPM (counts per million mapped reads) value of at least 0.5 in at least three libraries. Counts were converted to log2CPM, TMM normalized and precision weighted with the voom function of the limma package $(56,57)$. A linear model was fitted to each gene and empirical Bayes moderated t-statistics were used to assess differences in expression (58). Genes were called differentially expressed if they achieved a false discovery rate of 0.05 or less. Differential gene expression analysis, GO and KEGG pathway enrichment analysis were done using the $\mathrm{R} /$ Bioconductor package edgeR (59) and limma (57).

\section{DATA AVAILABILITY STATEMENT}

The raw data supporting the conclusions of this article will be made available by the authors, without undue reservation, to any qualified researcher.

\section{ETHICS STATEMENT}

The animal study was reviewed and approved by The Walter and Eliza Hall Institute Animal Ethics Committee.

\section{AUTHOR CONTRIBUTIONS}

RD, WG, SH-Z, RH, JR, FS-F-G, and MD performed experiments. RD, FS-F-G, and $\mathrm{NH}$ designed experiments and analyzed the data. RD, GG, JN, FS-F-G, MD, and $\mathrm{NH}$ contributed intellectual input and helped to interpret data. $\mathrm{NH}$ led the research program. $\mathrm{RD}$ and $\mathrm{NH}$ wrote the manuscript.

\section{FUNDING}

This work was supported in Australia by project grants from the National Health and Medical Research Council (NHMRC) of Australia (\#1124784, \#1066770, \#1057852, \#1124907 to NH; and \#1140406 to FS-F-G). NH was a NHMRC CDF2 Fellow (1124788), a recipient of a Melanoma Research Grant from the Harry J. Lloyd Charitable Trust, Melanoma Research Alliance Young Investigator Award, Ian Potter Foundation equipment grant and a CLIP grant from Cancer Research Institute. RD was a recipient of a Leukemia Foundation PhD scholarship, was supported by a Cancer Council Victoria postdoctoral fellowship and is currently a NHMRC Early Career Fellow (GNT1158615). FS-F-G was supported by a NHMRC Early Career Fellowship (1088703), a National Breast Cancer Foundation (NBCF) Fellowship (PF-15-008), a grant \#1120725 awarded through the Priority-driven Collaborative Cancer Research Scheme and funded by Cure Cancer Australia with the assistance of Cancer Australia. GG was supported by a post-doctoral fellowship from the Fondation ARC pour la recherche sur le Cancer and was currently supported by the Janssen Horizon Fonds de dotation. JN's lab was supported by the Groupement des Entreprises Françaises dans la Lutte contre le Cancer (GEFLUC) Marseille-Provence.

\section{ACKNOWLEDGMENTS}

We wish to thank Alison Campbell for technical assistance, Robert Schreiber for the MCA1956 cells and Tania Camilleri for excellent animal husbandry. We are grateful to the staff of the WEHI Bioservices, Monoclonal antibody facility, Flow cytometry facility and Clinical Translational Center. This study was made possible through Victorian State Government Operational Infrastructure Support and Australian Government NHMRC Independent Research Institute Infrastructure Support scheme.

\section{SUPPLEMENTARY MATERIAL}

The Supplementary Material for this article can be found online at: https://www.frontiersin.org/articles/10.3389/fimmu. 2020.00075/full\#supplementary-material 


\section{REFERENCES}

1. Huntington ND, Vosshenrich CA, Di Santo JP. Developmental pathways that generate natural-killer-cell diversity in mice and humans. Nat Rev Immunol. (2007) 7:703-14. doi: 10.1038/nri2154

2. Imai K, Matsuyama S, Miyake S, Suga K, Nakachi K. Natural cytotoxic activity of peripheral-blood lymphocytes and cancer incidence: an 11year follow-up study of a general population. Lancet. (2000) 356:1795-9. doi: 10.1016/S0140-6736(00)03231-1

3. Orange JS. Natural killer cell deficiency. J Allergy Clin Immunol. (2013) 132:515-25. doi: 10.1016/j.jaci.2013.07.020

4. Sathe P, Delconte RB, Souza-Fonseca-Guimaraes F, Seillet C, Chopin $\mathrm{M}$, Vandenberg $\mathrm{CJ}$, et al. Innate immunodeficiency following genetic ablation of Mcl1 in natural killer cells. Nat Commun. (2014) 5:4539. doi: 10.1038/ncomms5539

5. Souza-Fonseca-Guimaraes F, Cursons J, Huntington ND. The emergence of natural killer cells as a major target in cancer immunotherapy. Trends Immunol. (2019) 40:142-58. doi: 10.1016/j.it.2018.12.003

6. Barkholt L, Alici E, Conrad R, Sutlu T, Gilljam M, Stellan B, et al. Safety analysis of ex vivo-expanded NK and NK-like $\mathrm{T}$ cells administered to cancer patients: a phase I clinical study. Immunotherapy. (2009) 1:753-64. doi: 10.2217/imt.09.47

7. Mehta RS, Rezvani K. Chimeric antigen receptor expressing natural killer cells for the immunotherapy of cancer. Front Immunol. (2018) 9:283. doi: 10.3389/fimmu.2018.00283

8. Shimabukuro-Vornhagen A, Godel P, Subklewe M, Stemmler HJ, Schlosser HA, Schlaak M, et al. Cytokine release syndrome. J Immunother Cancer. (2018) 6:56. doi: 10.1186/s40425-018-0343-9

9. Delconte RB, Kolesnik TB, Dagley LF, Rautela J, Shi W, Putz EM, et al. CIS is a potent checkpoint in NK cell-mediated tumor immunity. Nat Immunol. (2016) 17:816-24. doi: 10.1038/ni.3470

10. Huntington ND. The unconventional expression of IL-15 and its role in NK cell homeostasis. Immunol Cell Biol. (2014) 92:210-3. doi: 10.1038/icb.2014.1

11. Rautela J, Huntington ND. IL-15 signaling in NK cell cancer immunotherapy. Curr Opin Immunol. (2017) 44:1-6. doi: 10.1016/j.coi.2016.10.004

12. Lucas M, Schachterle W, Oberle K, Aichele P, Diefenbach A. Dendritic cells prime natural killer cells by trans-presenting interleukin 15. Immunity. (2007) 26:503-17. doi: 10.1016/j.immuni.2007.03.006

13. Mortier E, Advincula R, Kim L, Chmura S, Barrera J, Reizis B, et al. Macrophage- and dendritic-cell-derived interleukin-15 receptor alpha supports homeostasis of distinct CD8+ T cell subsets. Immunity. (2009) 31:811-22. doi: 10.1016/j.immuni.2009.09.017

14. Chaix J, Tessmer MS, Hoebe K, Fuseri N, Ryffel B, Dalod M, et al. Cutting edge: Priming of NK cells by IL-18. J Immunol. (2008) 181:1627-31. doi: 10.4049/jimmunol.181.3.1627

15. Guia S, Cognet C, de Beaucoudrey L, Tessmer MS, Jouanguy E, Berger C, et al. A role for interleukin-12/23 in the maturation of human natural killer and CD56+ T cells in vivo. Blood. (2008) 111:5008-16. doi: 10.1182/blood-2007-11-122259

16. Sun JC, Madera S, Bezman NA, Beilke JN, Kaplan MH, Lanier LL. Proinflammatory cytokine signaling required for the generation of natural killer cell memory. J Exp Med. (2012) 209:947-54. doi: 10.1084/jem.201 11760

17. Ganal SC, Sanos SL, Kallfass C, Oberle K, Johner C, Kirschning C, et al. Priming of natural killer cells by nonmucosal mononuclear phagocytes requires instructive signals from commensal microbiota. Immunity. (2012) 37:171-86. doi: 10.1016/j.immuni.2012.05.020

18. Huntington ND, Tabarias H, Fairfax K, Brady J, Hayakawa Y, DegliEsposti MA, et al. NK cell maturation and peripheral homeostasis is associated with KLRG1 up-regulation. J Immunol. (2007) 178:4764-70. doi: 10.4049/jimmunol.178.8.4764

19. Chiossone L, Chaix J, Fuseri N, Roth C, Vivier E, Walzer T. Maturation of mouse NK cells is a 4-stage developmental program. Blood. (2009) 113:548896. doi: 10.1182/blood-2008-10-187179

20. Hayakawa Y, Smyth MJ. CD27 dissects mature NK cells into two subsets with distinct responsiveness and migratory capacity. J Immunol. (2006) 176:151724. doi: 10.4049/jimmunol.176.3.1517
21. Martinet L, Ferrari De Andrade L, Guillerey C, Lee JS, Liu J, Souza-FonsecaGuimaraes F, et al. DNAM-1 expression marks an alternative program of NK cell maturation. Cell Rep. (2015) 11:85-97. doi: 10.1016/j.celrep.2015.03.006

22. Kim S, Poursine-Laurent J, Truscott SM, Lybarger L, Song YJ, Yang L, et al. Licensing of natural killer cells by host major histocompatibility complex class I molecules. Nature. (2005) 436:709-13. doi: 10.1038/nature03847

23. Orr MT, Murphy WJ, Lanier LL. 'Unlicensed' natural killer cells dominate the response to cytomegalovirus infection. Nat Immunol. (2010) 11:321-7. doi: 10.1038/ni.1849

24. Belanger S, Tu MM, Rahim MM, Mahmoud AB, Patel R, Tai $\mathrm{LH}$, et al. Impaired natural killer cell self-education and "missingself" responses in Ly49-deficient mice. Blood. (2012) 120:592-602. doi: 10.1182/blood-2012-02-408732

25. Scholzen T, Gerdes J. The Ki-67 protein: from the known and the unknown. J Cell Physiol. (2000) 182:311-22. doi: 10.1002/(SICI)10974652(200003)182:3<311::AID-JCP1>3.0.CO;2-9

26. Jamieson AM, Isnard P, Dorfman JR, Coles MC, Raulet DH. Turnover and proliferation of NK cells in steady state and lymphopenic conditions. $J$ Immunol. (2004) 172:864-70. doi: 10.4049/jimmunol.172.2.864

27. Anthony SM, Rivas SC, Colpitts SL, Howard ME, Stonier SW, Schluns KS. Inflammatory signals regulate IL-15 in response to lymphodepletion. $J$ Immunol. (2016) 196:4544-52. doi: 10.4049/jimmunol.1600219

28. Fehniger TA, Suzuki K, Ponnappan A, VanDeusen JB, Cooper MA, Florea SM, et al. Fatal leukemia in interleukin 15 transgenic mice follows early expansions in natural killer and memory phenotype CD8+ T cells. J Exp Med. (2001) 193:219-31. doi: 10.1084/jem.193.2.219

29. Gattinoni L, Finkelstein SE, Klebanoff CA, Antony PA, Palmer DC, Spiess PJ, et al. Removal of homeostatic cytokine sinks by lymphodepletion enhances the efficacy of adoptively transferred tumor-specific CD8+ T cells. J Exp Med. (2005) 202:907-12. doi: 10.1084/jem.20050732

30. Alvarez M, Bouchlaka MN, Sckisel GD, Sungur CM, Chen M, Murphy WJ. Increased antitumor effects using IL-2 with anti-TGF-beta reveals competition between mouse NK and CD8 T cells. J Immunol. (2014) 193:1709-16. doi: 10.4049/jimmunol.1400034

31. Huntington ND, Puthalakath H, Gunn P, Naik E, Michalak EM, Smyth MJ, et al. Interleukin 15-mediated survival of natural killer cells is determined by interactions among Bim, Noxa and Mcl-1. Nat Immunol. (2007) 8:856-63. doi: $10.1038 /$ ni1 1487

32. Viant C, Guia S, Hennessy RJ, Rautela J, Pham K, Bernat C, et al. Cell cycle progression dictates the requirement for BCL2 in natural killer cell survival. $J$ Exp Med. (2017) 214:491-510. doi: 10.1084/jem.20160869

33. Vitale M, Cantoni C, Pietra G, Mingari MC, Moretta L. Effect of tumor cells and tumor microenvironment on NK-cell function. Euro J Immunol. (2014) 44:1582-92. doi: 10.1002/eji.201344272

34. Ferrari de Andrade L, Ngiow SF, Stannard K, Rusakiewicz S, Kalimutho M, Khanna KK, et al. Natural killer cells are essential for the ability of BRAF inhibitors to control BRAFV600E-mutant metastatic melanoma. Cancer Res. (2014) 74:7298-308. doi: 10.1158/0008-5472.CAN-14-1339

35. Quemeneur L, Gerland LM, Flacher M, Ffrench M, Revillard JP, Genestier L. Differential control of cell cycle, proliferation, and survival of primary T lymphocytes by purine and pyrimidine nucleotides. J Immunol. (2003) 170:4986-95. doi: 10.4049/jimmunol.170.10.4986

36. Villarino AV, Sciume G, Davis FP, Iwata S, Zitti B, Robinson GW, et al. Subset- and tissue-defined STAT5 thresholds control homeostasis and function of innate lymphoid cells. J Exp Med. (2017) 214:2999-3014. doi: 10.1084/jem.20150907

37. Lodolce JP, Boone DL, Chai S, Swain RE, Dassopoulos T, Trettin S, et al. IL-15 receptor maintains lymphoid homeostasis by supporting lymphocyte homing and proliferation. Immunity. (1998) 9:669-76. doi: 10.1016/S1074-7613(00)80664-0

38. Ranson T, Vosshenrich CA, Corcuff E, Richard O, Muller W, Di Santo JP. IL15 is an essential mediator of peripheral NK-cell homeostasis. Blood. (2003) 101:4887-93. doi: 10.1182/blood-2002-11-3392

39. Lopez-Verges S, Milush JM, Schwartz BS, Pando MJ, Jarjoura J, York VA, et al. Expansion of a unique CD57(+)NKG2Chi natural killer cell subset during acute human cytomegalovirus infection. Proc Natl Acad Sci USA. (2011) 108:14725-32. doi: 10.1073/pnas.1110900108 
40. Sun JC, Beilke JN, Lanier LL. Adaptive immune features of natural killer cells. Nature. (2009) 457:557-61. doi: 10.1038/nature07665

41. Bouillet P, Metcalf D, Huang DC, Tarlinton DM, Kay TW, Kontgen F, et al. Proapoptotic Bcl-2 relative Bim required for certain apoptotic responses, leukocyte homeostasis, and to preclude autoimmunity. Science. (1999) 286:1735-8. doi: 10.1126/science.286.5445.1735

42. Khattri R, Auger JA, Griffin MD, Sharpe AH, Bluestone JA. Lymphoproliferative disorder in CTLA-4 knockout mice is characterized by CD28-regulated activation of Th2 responses. J Immunol. (1999) 162:5784-91.

43. Lima M. Aggressive mature natural killer cell neoplasms: from epidemiology to diagnosis. Orphanet J Rare Dis. (2013) 8:95. doi: 10.1186/1750-1172-8-95

44. Suzuki R, Suzumiya J, Nakamura S, Aoki S, Notoya A, Ozaki S, et al. Aggressive natural killer-cell leukemia revisited: large granular lymphocyte leukemia of cytotoxic NK cells. Leukemia. (2004) 18:763-70. doi: $10.1038 /$ sj.leu.2403262

45. Lopez-Soto A, Gonzalez S, Smyth MJ, Galluzzi L. Control of metastasis by NK cells. Cancer Cell. (2017) 32:135-54. doi: 10.1016/j.ccell.2017.06.009

46. Holmes TD, El-Sherbiny YM, Davison A, Clough SL, Blair GE, Cook GP. A human NK cell activation/inhibition threshold allows small changes in the target cell surface phenotype to dramatically alter susceptibility to NK cells. J Immunol. (2011) 186:1538-45. doi: 10.4049/jimmunol. 1000951

47. Ullberg M, Jondal M. Recycling and target binding capacity of human natural killer cells. J Exp Med. (1981) 153:615-28. doi: 10.1084/jem.153.3.615

48. Bhat R, Watzl C. Serial killing of tumor cells by human natural killer cells-enhancement by therapeutic antibodies. PLoS ONE. (2007) 2:e326. doi: 10.1371/journal.pone.0000326

49. Mangan MS, Melo-Silva CR, Luu J, Bird CH, Koskinen A, Rizzitelli A, et al. A pro-survival role for the intracellular granzyme B inhibitor Serpinb9 in natural killer cells during poxvirus infection. Immunol Cell Biol. (2017) 95:884-94. doi: 10.1038/icb.2017.59

50. Neri S, Mariani E, Meneghetti A, Cattini L, Facchini A. Calceinacetyoxymethyl cytotoxicity assay: standardization of a method allowing additional analyses on recovered effector cells and supernatants. Clin Diagn Lab Immunol. (2001) 8:1131-5. doi: 10.1128/CDLI.8.6.1131-1135.2001

51. Hawkins ED, Hommel M, Turner ML, Battye FL, Markham JF, Hodgkin PD. Measuring lymphocyte proliferation, survival and differentiation using CFSE time-series data. Nat Protoc. (2007) 2:2057-67. doi: 10.1038/nprot.2007.297

52. Andrews S. FastQC: A Quality Control Tool for High Throughput Sequence Data. (2010). Available online at: http://www.bioinformatics.babraham.ac.uk/ projects/fastqc. (accessed January 23, 2019).
53. Bolger AM, Lohse M, Usadel B. Trimmomatic: a flexible trimmer for Illumina sequence data. Bioinformatics. (2014) 30:2114-20. doi: 10.1093/bioinformatics/btu170

54. Dobin A, Davis CA, Schlesinger F, Drenkow J, Zaleski C, Jha S, et al. STAR: ultrafast universal RNA-seq aligner. Bioinformatics. (2013) 29:15-21. doi: 10.1093/bioinformatics/bts635

55. Liao Y, Smyth GK, Shi W. featureCounts: an efficient general purpose program for assigning sequence reads to genomic features. Bioinformatics. (2014) 30:923-30. doi: 10.1093/bioinformatics/btt656

56. Law CW, Chen Y, Shi W, Smyth GK. voom: precision weights unlock linear model analysis tools for RNA-seq read counts. Genome Biol. (2014) 15:R29. doi: 10.1186/gb-2014-15-2-r29

57. Ritchie ME, Phipson B, Wu D, Hu Y, Law CW, Shi W, et al. limma powers differential expression analyses for RNA-sequencing and microarray studies. Nucleic Acids Res. (2015) 43:e47. doi: 10.1093/nar/g kv007

58. Smyth GK. Linear models and empirical bayes methods for assessing differential expression in microarray experiments. Stat Appl Genet Mol Biol. (2004) 3:1027. doi: 10.2202/1544-61 15.1027

59. Robinson MD, McCarthy DJ, Smyth GK. edgeR: a Bioconductor package for differential expression analysis of digital gene expression data. Bioinformatics. (2010) 26:139-40. doi: 10.1093/bioinformatics/btp616

Conflict of Interest: JR and NH are founders and shareholders of oNKo-Innate Pty Ltd. NH receives research funding from Servier and Anaxis Pharma Pty Ltd. $\mathrm{NH}$ and FS-F-G have a funded research collaborative agreement with Paranta Biosciences Ltd.

The remaining authors declare that the research was conducted in the absence of any commercial or financial relationships that could be construed as a potential conflict of interest.

Copyright (c) 2020 Delconte, Guittard, Goh, Hediyeh-Zadeh, Hennessy, Rautela, Davis, Souza-Fonseca-Guimaraes, Nunès and Huntington. This is an open-access article distributed under the terms of the Creative Commons Attribution License (CC $B Y)$. The use, distribution or reproduction in other forums is permitted, provided the original author(s) and the copyright owner(s) are credited and that the original publication in this journal is cited, in accordance with accepted academic practice. No use, distribution or reproduction is permitted which does not comply with these terms. 